\title{
A greedy algorithm for nonlinear inverse problems with an application to nonlinear inverse gravimetry
}

\author{
Max Kontak · Volker Michel
}

Received: date / Accepted: date

\begin{abstract}
Based on the Regularized Functional Matching Pursuit (RFMP) algorithm for linear inverse problems, we present an analogous iterative greedy algorithm for nonlinear inverse problems, called RFMP_NL. In comparison to established methods for nonlinear inverse problems, the algorithm is able to combine very diverse types of basis functions, for example, localized and global functions. This is important, in particular, in geoscientific applications, where global structures have to be distinguished from local anomalies. Furthermore, in contrast to other methods, the algorithm does not require the solution of large linear systems. We apply the RFMP_NL to the nonlinear inverse problem of gravimetry, where gravitational data are inverted for the shape of the surface or inner layer boundaries of planetary bodies. This inverse problem is described by a nonlinear integral operator, for which we additionally provide the Fréchet derivative. Finally, we present two synthetic numerical examples to show that it is beneficial to apply the presented method to inverse gravimetric problems.
\end{abstract}

Keywords greedy algorithm $\cdot$ inverse gravimetry $\cdot$ nonlinear inverse problem $\cdot$ regularization

Mathematics Subject Classification (2010) $65 \mathrm{~J} 22 \cdot 65 \mathrm{R} 32 \cdot 35 \mathrm{R} 30 \cdot 45 \mathrm{Q} 05$

\section{Introduction}

Nonlinear inverse problems arise in many fields, for example, in geosciences, medical imaging, or industrial applications. In this paper, we are particularly interested in the nonlinear

\section{Max Kontak}

Geomathematics Group, Department of Mathematics, University of Siegen, Walter-Flex-Str. 3, 57068 Siegen, Germany

Now at: High-Performance Computing, Simulation and Software Technology, DLR German Aerospace Center, Linder Höhe, 51147 Köln, Germany

E-mail: Max.Kontak@DLR.de

Volker Michel

Geomathematics Group, Department of Mathematics, University of Siegen, Walter-Flex-Str. 3, 57068 Siegen, Germany

E-mail: michel@mathematik.uni-siegen.de 
inverse gravimetric problem, which can be represented by a nonlinear integral equation involving a function on the sphere. The work at hand is based on the $\mathrm{PhD}$ thesis (Kontak 2018) by the first author.

We will provide a novel iterative algorithm for the solution of nonlinear inverse problems of the following form.

Problem 1 (Nonlinear inverse problem) Let $\mathscr{X}, \mathscr{Y}$ be Hilbert spaces and let the operator $\mathcal{S}: \mathscr{X} \rightarrow \mathscr{Y}$ be Fréchet differentiable and possibly nonlinear. For given data $g \in \mathscr{Y}$, find $f^{*} \in \mathscr{X}$ such that

$$
\mathcal{S}\left[f^{*}\right]=g .
$$

We will derive an algorithm that is called the Regularized Functional Matching Pursuit for nonlinear inverse problems (RFMP_NL). It is based on the Regularized Functional Matching Pursuit (RFMP), which has been presented and analyzed in (Berkel et al. 2011; Fischer and Michel 2012, 2013a,b; Gutting et al. 2017; Kontak and Michel 2018; Michel 2015; Michel and Orzlowski 2017; Michel and Telschow 2014). The RFMP is a greedy-type algorithm for linear inverse problems $\mathcal{T} f^{*}=g$, where $\mathcal{T}: \mathscr{X} \rightarrow \mathscr{Y}$ is a linear and bounded operator between Hilbert spaces $\mathscr{X}, \mathscr{Y}$, and $g \in \mathscr{Y}$ represents given data.

The idea behind this greedy algorithm is the following: we prescribe a so-called dictionary $\mathscr{D} \subseteq \mathscr{X}$, which can consist of an arbitrary collection of elements from $\mathscr{X}$. Starting with an initial approximation $f_{0} \in \mathscr{X}$ of the solution $f^{*} \in \mathscr{X}$, for $k=0,1,2, \ldots$, we iteratively choose the pair $\left(\alpha_{k+1}, d_{k+1}\right) \in \mathbb{R} \times \mathscr{D}$ that minimizes the Tikhonov functional

$$
\left\|g-\mathcal{T} f_{k}-\alpha_{k+1} \mathcal{T} d_{k+1}\right\|_{\mathscr{Y}}^{2}+\lambda\left\|f_{k}+\alpha_{k+1} d_{k+1}\right\|_{\mathscr{X}}^{2}
$$

for the given previous iteration $f_{k} \in \mathscr{X}$ and a prescribed regularization parameter $\lambda>0$.

Since the dictionary $\mathscr{D}$ does not need to form a basis, the algorithm is able to combine very diverse types of elements from the Hilbert space $\mathscr{X}$, for example, both global and localized functions if $\mathscr{X}$ is a function space. The numerical results in the previously mentioned references show that this property of the RFMP makes it beneficial in comparison to other methods for linear inverse problems. These are often more easy to formulate in the Hilbert space setting, but when it comes to the implementation one has to stick to one specific basis system.

Based on the RFMP, two other algorithms for linear inverse problems have previously been developed. The Regularized Orthogonal Functional Matching Pursuit (ROFMP) (see Michel and Telschow 2016; Telschow 2014) overcomes the difficulty that the RFMP may choose a single dictionary element multiple times, which is not optimal, by an orthogonalization procedure in every iteration step. Numerical results show that the ROFMP is very suitable, in particular, for those geoscientific inverse problems, where only very scattered data are given. The Regularized Weak Functional Matching Pursuit (RWFMP) (see Kontak 2018; Kontak and Michel 2018) removes several open problems in the theoretical analysis of the RFMP, for example, a convergence rate for the regularized case could be derived and the convergence in arbitrary Hilbert spaces was proved. Additionally, numerical examples show that the RWFMP accelerates the iteration of the RFMP by several factors by using a specific search strategy to find the next pair $\left(\alpha_{k+1}, d_{k+1}\right)$.

Other iterative methods for nonlinear inverse problems are, for example, the LevenbergMarquardt method (see Levenberg 1944; Marquardt 1963) and the iteratively regularized Gauß-Newton method (see Bakushinsky 1992), and many more. In most of these methods, one has to solve large linear systems in every iteration, which is not the case for the newly presented algorithm. Additionally, one has to stick to one single specific basis system in the 
implementation to ensure the regularity of the arising matrices, which is not necessary for the RFMP_NL. A detailed comparison of the new greedy algorithm to existing methods for nonlinear inverse problems will be given in Section 5 of this paper.

As we have already mentioned, the nonlinear inverse problem, which we will deal with, is the nonlinear inverse gravimetric problem. In contrast to the linear inverse gravimetric problem, which is concerned with the inversion of the gravitational potential for the mass density distribution inside a given body of mass, the nonlinear problem is the inversion of the potential for the shape of the body of mass given a mass density model. Nowadays, the gravitational field of the Earth is measured by satellite missions like CHAMP (see Reigber et al. 1999), GRACE (see Tapley et al. 2004), GOCE (see Drinkwater et al. 2003), as well as the upcoming GRACE follow-on mission (see Flechtner et al. 2014). The nonlinear inverse gravimetric problem can be used to determine the shape of the Earth from these satellite measurements. Since this can be done with a (likely) higher precision by radar technology, it is more important that gravity inversion also enables us to study the boundaries between different layers of the Earth, for example, the Mohorovičić discontinuity, which is the boundary between the crust and the mantle (see, for example, Clauser 2014, Section 1.5). Satellite missions to the Moon (GRAIL, see Zuber et al. 2013) and Jupiter (Juno, see Bolton et al. 2017; Matousek 2007) also allow for the study of the interior of these celestial bodies.

The paper is structured as follows. In Section 2, we will provide the necessary basics of the notation and of spherical geometry that we will need in the paper. Section 3 is dedicated to inverse gravimetry. We will present both the linear and nonlinear inverse gravimetric problem and for the latter, we will provide the Fréchet derivative of the associated operator. In Section 4, we will derive the RFMP_NL, which we will compare to other methods for nonlinear inverse problems in Section 5. Section 6 consists of two numerical examples for the application of the RFMP_NL to the nonlinear inverse gravimetric problem. We finally give a conclusion and an outlook in Section 7.

\section{Basics}

In this section, we will briefly summarize the basic notation that we will need in this paper, in particular, the basics of spherical geometry.

By

$$
\mathbb{S}^{2}:=\left\{x \in \mathbb{R}^{3}|| x \mid=1\right\}
$$

we denote the unit sphere in $\mathbb{R}^{3}$, where $|\cdot|$ represents the usual Euclidean norm. It is wellknown that every point $x \in \mathbb{R}^{3}$ can be described by the polar coordinates

$$
x(r, \varphi, t)=\left(\begin{array}{c}
r \sqrt{1-t^{2}} \cos \varphi \\
r \sqrt{1-t^{2}} \sin \varphi \\
r t
\end{array}\right)
$$

where $r \in[0, \infty), \varphi \in[0,2 \pi)$, and $t \in[-1,1]$. Analogously, every point $\xi \in \mathbb{S}^{2}$ can be described by

$$
\xi(\varphi, t)=\left(\begin{array}{c}
\sqrt{1-t^{2}} \cos \varphi \\
\sqrt{1-t^{2}} \sin \varphi \\
t
\end{array}\right)
$$


where $\varphi \in[0,2 \pi)$ and $t \in[-1,1]$

Both on subsets $U \subseteq \mathbb{R}^{3}$ and the sphere $\mathbb{S}^{2}$, we define the spaces of real-valued continuous functions $\mathrm{C}(M)$ and of $k$-times continuously differentiable functions $\mathrm{C}^{(k)}(M)$, where $M \in\left\{U, \mathbb{S}^{2}\right\}$ with the corresponding norms $\|\cdot\|_{\mathrm{C}(M)}$ and $\|\cdot\|_{\mathrm{C}^{(k)}(M)}$, respectively.

For measurable functions $f: U \rightarrow \mathbb{R}, g: \mathbb{S}^{2} \rightarrow \mathbb{R}$ the integrals

$$
\int_{U} f(x) \mathrm{d} x, \quad \quad \int_{\mathbb{S}^{2}} g(\xi) \mathrm{d} \omega(\xi),
$$

denote the integral of $f$ with respect to the Lebesgue measure on $U$ and the integral of $g$ with respect to the surface measure on $\mathbb{S}^{2}$, respectively. Using polar coordinates, we can decompose any integral over the space $\mathbb{R}^{3}$ into a radial and a spherical part such that

$$
\int_{\mathbb{R}^{3}} f(x) \mathrm{d} x=\int_{\mathbb{S}^{2}} \int_{0}^{\infty} f(r \xi) r^{2} \mathrm{~d} r \mathrm{~d} \omega(\xi)
$$

for $f: \mathbb{R}^{3} \rightarrow \mathbb{R}$.

Using these integrals, we can define the norms

$$
\begin{aligned}
\|f\|_{\mathrm{L}^{2}(U)} & :=\left(\int_{U}|f(x)|^{2} \mathrm{~d} x\right)^{1 / 2}, \\
\|g\|_{\mathrm{L}^{2}\left(\mathbb{S}^{2}\right)} & :=\left(\int_{\mathbb{S}^{2}}|g(\xi)|^{2} \mathrm{~d} \omega(\xi)\right)^{1 / 2},
\end{aligned}
$$

for measurable functions $f: U \rightarrow \mathbb{R}, g: \mathbb{S}^{2} \rightarrow \mathbb{R}$. The $\mathrm{L}^{2}(U)$ - and $\mathrm{L}^{2}\left(\mathbb{S}^{2}\right)$-spaces are consequently given as

$$
\begin{aligned}
\mathrm{L}^{2}(U) & :=\left\{f: U \rightarrow \mathbb{R} \mid f \text { is measurable and }\|f\|_{\mathrm{L}^{2}(U)}<\infty\right\} . \\
\mathrm{L}^{2}\left(\mathbb{S}^{2}\right) & :=\left\{g: \mathbb{S}^{2} \rightarrow \mathbb{R} \mid g \text { is measurable and }\|g\|_{\mathrm{L}^{2}\left(\mathbb{S}^{2}\right)}<\infty\right\},
\end{aligned}
$$

where we, as usual, formally identify functions with each other, which are equal almost everywhere.

It is well-known that $\mathrm{L}^{2}(U)$ and $\mathrm{L}^{2}\left(\mathbb{S}^{2}\right)$ are Hilbert spaces with the corresponding inner product

$$
\begin{aligned}
\left\langle f_{1}, f_{2}\right\rangle_{\mathrm{L}^{2}(U)} & :=\int_{U} f_{1}(x) f_{2}(x) \mathrm{d} x, \\
\left\langle g_{1}, g_{2}\right\rangle_{\mathrm{L}^{2}\left(\mathbb{S}^{2}\right)} & :=\int_{\mathbb{S}^{2}} g_{1}(\xi) g_{2}(\xi) \mathrm{d} \omega(\xi),
\end{aligned}
$$

where $f_{1}, f_{2} \in \mathrm{L}^{2}(U)$ and $g_{1}, g_{2} \in \mathrm{L}^{2}\left(\mathbb{S}^{2}\right)$.

The system of (fully normalized) spherical harmonics is given by

$$
\left\{Y_{n, j} \mid n \in \mathbb{N}_{0}, j=-n, \ldots, n\right\},
$$

where

$$
\begin{aligned}
Y_{n, j}(\xi(\varphi, t)):= & \sqrt{\frac{(2 n+1)(n-|j|) !\left(2-\delta_{j 0}\right)}{4 \pi(n+|j|) !}} P_{n,|j|}(t) \\
& \times \begin{cases}\sin (j \varphi), & j=1, \ldots, n, \\
\cos (j \varphi), & j=-n, \ldots, 0,\end{cases}
\end{aligned}
$$


and for $n \in \mathbb{N}_{0}, m=0, \ldots, n$,

$$
P_{n, m}(t):=\left(1-t^{2}\right)^{m / 2} \frac{\mathrm{d}^{m}}{\mathrm{~d} t^{m}} P_{n}(t), \quad t \in[-1,1],
$$

are the associated Legendre functions with the Legendre polynomials $P_{n, 0}=P_{n}$. It is wellknown that this system is a complete orthonormal system in $\mathrm{L}^{2}\left(\mathbb{S}^{2}\right)$. Furthermore, it is known that all spherical harmonics are not localized on any subset of the sphere such that they are an ideal choice for the approximation of functions with global structures on the sphere.

Moreover, we define the function $Q_{h}:[-1,1] \rightarrow \mathbb{R}, h \in(0,1)$,

$$
Q_{h}(t):=\frac{1}{4 \pi} \frac{1-h^{2}}{\left(1+h^{2}-2 h t\right)^{3 / 2}},
$$

such that the kernel $\mathbb{S}^{2} \times \mathbb{S}^{2} \ni(\xi, \eta) \mapsto Q_{h}(\xi \cdot \eta)$ is called the Abel-Poisson kernel.

The Abel-Poisson kernel is a zonal function, that is, for fixed $\xi \in \mathbb{S}^{2}$ the function $Q_{h, \xi}(\eta):=Q_{h}(\xi \cdot \eta)$ does only depend on the distance (or angle) between $\xi$ and $\eta$. Furthermore, it can be shown that for $h \nearrow 1$, the latter function is more and more concentrated at the point $\xi \in \mathbb{S}^{2}$, which makes it predestined for the local approximation of functions.

In our numerical examples for a nonlinear inverse problem, we will combine both spherical harmonics and Abel-Poisson kernels to account for both global and local structures in the solution.

\section{Nonlinear inverse gravimetry}

Inverse gravimetry is concerned with the gathering of information about the shape $\mathcal{E} \subseteq \mathbb{R}^{3}$ and the mass density $\rho: \mathcal{E} \rightarrow \mathbb{R}^{3}$ from the gravitational potential $U_{\mathcal{E}, \rho}(y): \mathbb{R}^{3} \backslash \overline{\mathcal{E}} \rightarrow \mathbb{R}$,

$$
U_{\mathcal{E}, \rho}(y):=\int_{\mathcal{E}} \frac{\rho(x)}{|x-y|} \mathrm{d} x,
$$

where, for simplicity, we omit the gravitational constant. We assume that $\mathcal{E}$ is a bounded open domain in $\mathbb{R}^{3}$ with a piecewise smooth boundary and that the mass density function $\rho$ is measurable and bounded. Note that we allow negative values of the mass density function, which is not reasonable from the physical perspective. However, it turns out that the relation between anomalies of the mass density and those of the gravitational potential can also be described by the integral in (2) such that, in this case, negative values for $\rho$ may occur.

Under these presumptions, it is well known that the gravitational potential is harmonic outside the set $\overline{\mathcal{E}}$ (see Mikhlin 1970, Theorem 11.6.2). Additionally, it satisfies the Poisson equation in $\mathcal{E}$ if $\rho$ is Lipschitz continuous (see Mikhlin 1970, Theorem 11.6.3).

In the functional analytic approach in Hilbert spaces, one often does not know whether $\rho$ is bounded. Instead, one assumes $\rho \in \mathrm{L}^{2}(\mathcal{E})$, which does not imply (essential) boundedness of $\rho$. The theory of Fredholm integral equations yields the following theorem (see, for example, Yosida 1980, Example 1 in Section VII.3, Example 2 in Section X.2).

Theorem 2 Let $\rho \in \mathrm{L}^{2}(\mathcal{E})$ and a regular surface $S$ be given such that $\overline{\mathcal{E}} \subseteq S^{\mathrm{int}}$. Then we have $\left.U_{\mathcal{E}, \rho}\right|_{S} \in \mathrm{L}^{2}(S)$.

Furthermore, for fixed $\mathcal{E}$, the operator $\mathcal{T}_{\mathcal{E}}: \mathrm{L}^{2}(\mathcal{E}) \rightarrow \mathrm{L}^{2}(S),\left.\rho \mapsto U_{\mathcal{E}, \rho}\right|_{S}$ is a compact linear operator. 


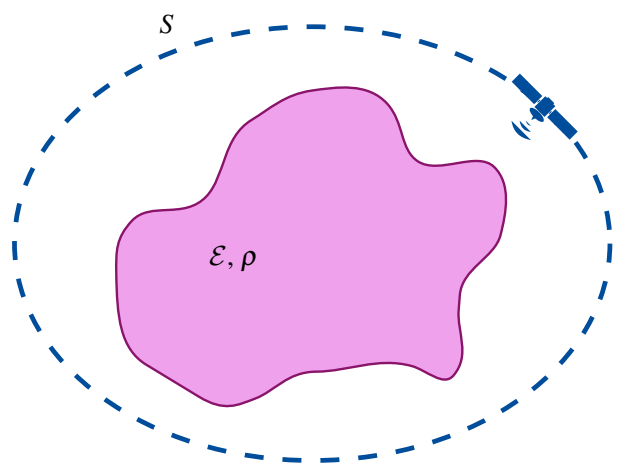

Figure 1: Two-dimensional sketch of the general inverse gravimetric problem: the body $\mathcal{E}$ is filled with mass of density $\rho$ (purple). The gravitational potential is measured (for example by a satellite) on a regular surface $S$ (blue).

Here, sticking to Freeden and Michel (2004, Section 3.1.1), we call a surface $\Sigma \subseteq \mathbb{R}^{3}$ a regular surface if it satisfies:

1. the space $\mathbb{R}^{3}$ is divided into a bounded region $\Sigma^{\text {int }}$ and an unbounded region $\Sigma^{\text {ext }}$ such that

$$
\Sigma^{\mathrm{ext}}=\mathbb{R}^{3} \backslash \overline{\Sigma^{\mathrm{int}}}, \quad \Sigma=\overline{\Sigma^{\mathrm{int}}} \cap \overline{\Sigma^{\mathrm{ext}}}, \quad \Sigma^{\mathrm{int}} \cap \Sigma^{\mathrm{ext}}=\emptyset,
$$

2. $\Sigma^{\text {int }}$ contains the origin,

3. $\Sigma$ is a closed and compact surface, which is free of double points,

4. $\Sigma$ has a continuously differentiable outer unit normal field $v: \Sigma \rightarrow \mathbb{R}^{3}$.

In consequence, Green's identities and Gauß' law are valid in $\Sigma^{\text {int }}$.

We will consider the inverse problem of gravimetry in the following form.

Problem 3 (General inverse gravimetric problem) Let $S \subseteq \mathbb{R}^{3}$ be a regular surface and $g \in \mathrm{L}^{2}(S)$ be a given function. Find $\mathcal{E} \subseteq S^{\text {int }}$ and $\rho \in \mathrm{L}^{2}(\mathcal{E})$ such that

$$
\left.U_{\mathcal{E}, \rho}\right|_{S}=g
$$

The situation is sketched in Figure 1. It is well-known that the gravitational potential of two balls of radii $R_{1}, R_{2}>0$ is the same in all points $y \in \mathbb{R}^{3}$ with $|y|>\max \left\{R_{1}, R_{2}\right\}$ if the balls possess equal total masses. Thus, the determination of both $\mathcal{E}$ and $\rho$ uniquely from gravitational data fails already in a very elementary setting incorporating simple geometries.

Consequently, in most of the literature on inverse gravimetry it is assumed that either the domain $\mathcal{E}$ or the mass density function $\rho$ is known and the inverse problem is to find the respective other unknown, which leads to the linear and the nonlinear inverse problem, respectively. In the following, we will first briefly summarize some results about the linear inverse gravimetric problem, in particular, its difficulties regarding the uniqueness of a solution. Afterwards, we will derive the nonlinear inverse gravimetric problem, where there is no problem with a non-uniqueness of the solution. 
3.1 Linear inverse gravimetric problem

We obtain the linear inverse gravimetric problem from the general inverse gravimetric problem by assuming that the shape $\mathcal{E}$ of the Earth is known. This results in the following problem.

Problem 4 (Linear inverse gravimetric problem) Let $S \subseteq \mathbb{R}^{3}$ be a regular surface, let $\mathcal{E}$ be a bounded open domain such that $\overline{\mathcal{E}} \subseteq S^{\text {int }}$, and let a function $g \in \mathrm{L}^{2}(S)$ be given.

Find $\rho \in \mathrm{L}^{2}(\mathcal{E})$ such that

$$
\left.U_{\mathcal{E}, \rho}\right|_{S}=g
$$

The operator that maps $\rho \in \mathrm{L}^{2}(\mathcal{E})$ to $\left.U_{\mathcal{E}, \rho}\right|_{S}$ for fixed $\mathcal{E}$ is denoted by $\mathcal{T}_{\mathcal{E}}: \mathrm{L}^{2}(\mathcal{E}) \rightarrow \mathrm{L}^{2}(S)$ and the operator equation

$$
\mathcal{T}_{\mathcal{E}}(\rho)=g
$$

is called the linear inverse gravimetric problem.

In the following, we will discuss the ill-posedness of the inverse problem in (3), that is, the existence, uniqueness, and stability of a solution of (3). We start with the following wellknown result about the non-uniqueness of the solution, which was given in the following form in Weck (1972).

Theorem 5 (cf. Weck 1972, Lemma 1) The null space of the operator $\mathcal{T}_{\mathcal{E}}$ is given by

$$
\operatorname{null} \mathcal{T}_{\mathcal{E}}=\left\{\Delta f \mid f \in \mathrm{H}_{0}^{2}(\mathcal{E})\right\},
$$

where $\mathrm{H}_{0}^{2}(\mathcal{E})$ is the completion of the space of arbitrarily often differentiable functions with compact support in $\mathcal{E}$ with respect to the well-known $\mathrm{H}^{2}(\mathcal{E})$-Sobolev norm. Furthermore, the orthogonal complement of the null space is given by

$$
\left(\operatorname{null} \mathcal{T}_{\mathcal{E}}\right)^{\perp}=\operatorname{null} \Delta:=\left\{f \in \mathrm{C}^{(\infty)}(\mathcal{E}) \mid \Delta f=0\right\}
$$

that is, it consists of all harmonic functions.

Note that Weyl's Lemma (cf. Freeden and Gerhards 2013, Section 4.1.2) states that every harmonic distribution can be represented by a function, which leads to the use of the space $\mathrm{C}^{(\infty)}(\mathcal{E})$ in $(4)$. Note furthermore that the result in (4) had been proved before, see Lauricella (1912); Pizzetti $(1909,1910)$.

In consequence, one can obtain a unique solution of the linear inverse gravimetric problem if one imposes a harmonicity condition on $\rho$. Unfortunately, this condition on the density function lacks a physical interpretation (cf. Michel and Fokas 2008), since the maximum principle for harmonic functions states that the density would have to attain its maximum at the Earth's surface. This is not reasonable, at least, if the complete density distribution is to be determined, since the density inside the Earth arguably increases towards the center. However, for the investigation of density anomalies, for example, due to short-period temporal variations, which basically occur on the surface only, such a constraint can be justified. In Michel and Fokas (2008), several other conditions on the density are presented, which also yield a unique solution. These considerations are generalized in Leweke et al. (2018) to a certain class of integral equations. This also includes the physically relevant case of a surface density. 
Concerning the existence and the stability, we can refer to the theory of compact operators. Since $\mathcal{T}_{\mathcal{E}}$ is compact, there exists a singular system $\left(\sigma_{j}, f_{j}, g_{j}\right)_{j \in \mathbb{N}_{0}}$ and in terms of this singular system a necessary and sufficient condition for the existence of a solution of (3) for given data $g \in \mathrm{L}^{2}(S)$ is the Picard condition

$$
\sum_{j=1}^{\infty} \frac{1}{\sigma_{j}^{2}}\left|\left\langle g, g_{j}\right\rangle\right|^{2}<\infty
$$

(see, for example, Engl et al. 1996, Theorem 2.8). It is also well-known that every compact operator in infinite-dimensional spaces has an unbounded inverse (see, for example, Rieder 2003, Satz 2.2.8(e)) such that we obtain the instability of the inverse problem.

Currently, the linear inverse gravimetric problem is relevant, in particular, where timedependent GRACE data are used to search for (climate-induced) mass transports at the Earth's surface. The nonlinear version is geophysically relevant for determining boundary layers, which are important for the understanding of geodynamic processes. We will see that it was proved that the solution of the nonlinear problem is both unique and stable.

\subsection{Nonlinear inverse gravimetric problem}

We have derived Problem 4 in the previous section by assuming that the shape of the Earth $\mathcal{E}$ is known in Problem 3. To obtain the nonlinear inverse gravimetric problem, we proceed the other way round. We assume that a model for the mass density function is available (for example, we could use PREM, see Dziewonski and Anderson 1981) such that we are concerned with the determination of the shape of the Earth. The corresponding inverse problem looks as follows.

Problem 6 (Nonlinear inverse gravimetric problem) Let $S \subseteq \mathbb{R}^{3}$ be a regular surface, let $\rho \in \mathrm{L}^{2}\left(S^{\mathrm{int}}\right)$ be a mass density model, and let a function $g \in \mathrm{L}^{2}(S)$ be given. Find $\mathcal{E}$ such that $\overline{\mathcal{E}} \subseteq S^{\text {int }}$ and

$$
\left.U_{\mathcal{E}, \rho}\right|_{S}=g
$$

The operator that maps $\mathcal{E} \subseteq S^{\mathrm{int}}$ to $\left.U_{\mathcal{E}, \rho}\right|_{S}$ for fixed $\rho$ is denoted by $\tilde{\mathcal{S}}_{\rho}$ and the operator equation

$$
\tilde{\mathcal{S}}_{\rho}[\mathcal{E}]=g
$$

is called the nonlinear inverse gravimetric problem.

Since the operator $\tilde{\mathcal{S}}_{\rho}$ should be formally defined on a space of subsets of $S^{\text {int }}$, which is quite difficult to handle, a popular approach in the literature is the restriction to star-shaped sets with respect to the origin. This, consequently, yields the following problem.

Problem 7 (Nonlinear inverse gravimetric problem, star-shaped) Let $S \subseteq \mathbb{R}^{3}$ be a regular surface, let $\rho \in \mathrm{L}^{2}\left(S^{\mathrm{int}}\right)$ be a mass density model, and let a function $g \in \overline{\mathrm{L}}^{2}(S)$ be given.

Find a function $\sigma: \mathbb{S}^{2} \rightarrow(0, \infty)$ such that $\mathcal{E}=\Sigma^{\text {int }}$, where the regular surface $\Sigma \subseteq S^{\text {int }}$ is given by

$$
\Sigma:=\left\{r \xi \in \mathbb{R}^{3} \mid \xi \in \mathbb{S}^{2}, r=\sigma(\xi)\right\},
$$




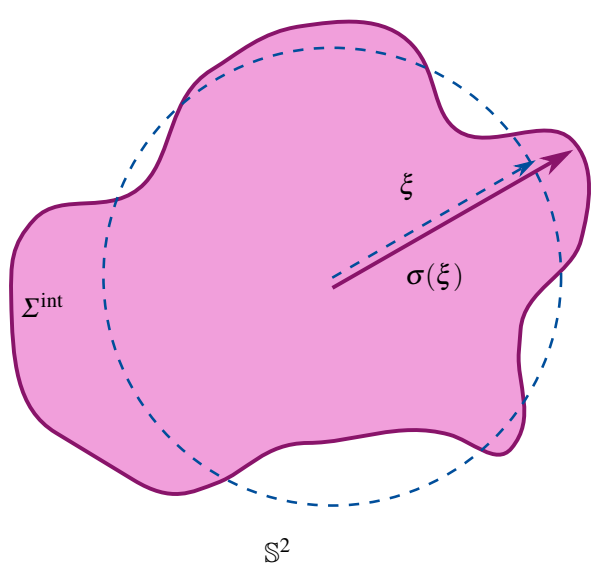

Figure 2: Geometrical representation of the situation in Problem 7 (two-dimensional slice): the boundary $\Sigma$ of the star-shaped body of mass $\Sigma^{\text {int }}$ is parametrized by a function $\sigma$ on $\mathbb{S}^{2}$.

and

$$
\left.U_{\Sigma^{\text {int }, \rho}}\right|_{S}=g .
$$

The operator that maps the function $\sigma$ to $\left.U_{\Sigma^{\text {int }}, \rho}\right|_{S}$ for fixed $\rho$ is denoted by $\mathcal{S}_{\rho}$ and the operator equation

$$
\mathcal{S}_{\rho}[\sigma]=g
$$

is called the nonlinear inverse gravimetric problem (with a star-shaped domain).

Note that, using polar coordinates, the nonlinear integral operator has the expression

$$
\begin{aligned}
\mathcal{S}_{\rho}[\sigma](y) & =U_{\Sigma^{\text {int }}, \rho}(y) \\
& =\int_{\Sigma^{\text {int }}} \frac{\rho(x)}{|x-y|} \mathrm{d} x \\
& =\int_{\mathbb{S}^{2}} \int_{0}^{\sigma(\xi)} \frac{\rho(r \xi)}{|r \xi-y|} r^{2} \mathrm{~d} r \mathrm{~d} \omega(\xi) .
\end{aligned}
$$

A sketch of the setting for Problem 7 can be found in Figure 2 .

The most extensive analysis of the uniqueness and stability of the nonlinear inverse gravimetric problem was accomplished in Isakov (1990), although there is a long list of references for earlier results on the topic (for example, Novikov 1938; Weck 1972). We only cite the uniqueness and stability result for constant density $\rho \equiv 1$ and refer the reader to the book Isakov (1990) for more general results. We start with the uniqueness result.

Theorem 8 (cf. Isakov 1990, Theorem 2.2.1) Let $S \subseteq \mathbb{R}^{3}$ be a regular surface. Suppose that $\Sigma_{1}, \Sigma_{2} \subseteq S^{\text {int }}$ are regular surfaces such that $\Sigma_{1}^{\text {int }}, \Sigma_{2}^{\text {int }}$ are star-shaped.

If

$$
\left.U_{\Sigma_{1}^{\mathrm{int}}, 1}\right|_{S^{\mathrm{ext}}}=\left.U_{\Sigma_{2}^{\mathrm{int}}, 1}\right|_{S^{\mathrm{ext}}}
$$

then $\Sigma_{1}=\Sigma_{2}$. 
Furthermore, we have the following stability estimate, where we restricted ourselves to the particular case of $S=\mathbb{S}^{2}$.

Theorem 9 (cf. Isakov 1990, Theorem 3.6.1) Let $S=\mathbb{S}^{2}$ and two regular surfaces $\Sigma_{1}, \Sigma_{2} \subseteq$ $S^{\text {int }}$ be given, which are parametrized by functions $\sigma_{1}, \sigma_{2}: \mathbb{S}^{2} \rightarrow(0,1)$ in the sense of (5) such that $\Sigma_{1}^{\mathrm{int}}, \Sigma_{2}^{\mathrm{int}}$ are star-shaped. Additionally, it is required that there exists a constant $h>0$ such that $\sigma_{1}(\xi), \sigma_{2}(\xi) \in(h, 1-h)$ for all $\xi \in \mathbb{S}^{2}$ and that $\sigma_{1}, \sigma_{2} \in \mathrm{C}^{(2)}\left(\mathbb{S}^{2}\right)$.

Then, there is a constant $C>0$ such that if

$$
|| \nabla U_{\Sigma_{1}^{\text {int }}, 1}(y)|-| \nabla U_{\Sigma_{2}^{\text {int }}, 1}(y)||<\varepsilon, \quad \text { for all } y \in S \text {, }
$$

then,

$$
\left|\sigma_{1}(\xi)-\sigma_{2}(\xi)\right|<C|\log \varepsilon|^{-1 / C}, \quad \text { for all } \xi \in \mathbb{S}^{2}
$$

Clearly, this is a stability estimate for the nonlinear inverse gravimetric problem, since it shows that the solution depends continuously on the data.

Unfortunately, the stability of the inverse problem can be described as being weak (cf. Isakov 2006, Section 1.1) due to the logarithmic nature of the estimate, which leads to "numerical difficulties" (Isakov 2006). This is the reason why, in practice, one still needs to apply a regularization technique although the nonlinear inverse gravimetric problem is stable in theory.

In conclusion, by addressing the nonlinear inverse problem of gravimetry instead of the linear one, the problem gets more difficult because of the nonlinearity. On the other hand, the solution of the nonlinear problem is both unique and stable (at least in theory), which is advantageous and beneficial for the numerical solution of the inverse problem.

We want to mention that one can also formulate the nonlinear inverse gravimetric problem as an inverse source problem for a partial differential equation, namely, the Poisson equation. This was, for example, done by Hettlich and Rundell (1996). Other inverse source problems of this type are related to the heat equation (see, for example, Hettlich and Rundell 1997) and the Helmholtz equation (see, for example, Elschner and Yamamoto 2006; Hettlich and Rundell 2000).

\subsection{The Gâteaux and Fréchet derivative of the nonlinear operator}

Most algorithms for nonlinear inverse problems use either the Fréchet or Gâteaux derivative of the involved operator. Therefore, we will first compute the Gâteaux derivative of the operator $\mathcal{S}_{\rho}$ both as an operator $\mathrm{C}\left(\mathbb{S}^{2}\right) \rightarrow \mathrm{C}(S)$ and $\mathrm{L}^{2}\left(\mathbb{S}^{2}\right) \rightarrow \mathrm{L}^{2}(S)$, where $S$ is a regular surface.

To compute the Gâteaux derivative, we will use the following special case of Leibniz' rule for differentiation of integrals (cf. Holmes 2009, Theorem 6.2).

Lemma 10 Let $f, g: \mathbb{R} \rightarrow \mathbb{R}$ be sufficiently smooth. Then

$$
\frac{\mathrm{d}}{\mathrm{d} t} \int_{0}^{g(t)} f(x) \mathrm{d} x=f(g(t)) g^{\prime}(t)
$$


The Gâteaux derivative $\mathcal{S}_{\rho}^{\prime}[\sigma](\tau): \mathrm{C}\left(\mathbb{S}^{2}\right) \rightarrow \mathrm{C}(S)$ at $\sigma \in \mathrm{C}\left(\mathbb{S}^{2}\right)$ in the direction $\tau \in$ $\mathrm{C}\left(\mathbb{S}^{2}\right)$ can now be obtained by an application of the preceding lemma to the expression in (6):

$$
\begin{aligned}
\mathcal{S}_{\rho}^{\prime}[\sigma](\tau)(y) & =\left.\frac{\mathrm{d}}{\mathrm{d} \varepsilon} \mathcal{S}_{\rho}[\sigma+\varepsilon \tau](y)\right|_{\varepsilon=0} \\
& \left.\sim \int_{\mathbb{S}^{2}} \frac{\mathrm{d}}{\mathrm{d} \varepsilon} \int_{0}^{\sigma(\xi)+\varepsilon \tau(\xi)} \frac{\rho(r \xi)}{|r \xi-y|} r^{2} \mathrm{~d} r\right|_{\varepsilon=0} \mathrm{~d} \omega(\xi) \\
& =\left.\int_{\mathbb{S}^{2}} \frac{\rho((\sigma(\xi)+\varepsilon \tau(\xi)) \xi)}{|(\sigma(\xi)+\varepsilon \tau(\xi)) \xi-y|}(\sigma(\xi)+\varepsilon \tau(\xi))^{2} \tau(\xi)\right|_{\varepsilon=0} \mathrm{~d} \omega(\xi) \\
& =\int_{\mathbb{S}^{2}} \frac{\rho(\sigma(\xi) \xi)}{|\sigma(\xi) \xi-y|}(\sigma(\xi))^{2} \tau(\xi) \mathrm{d} \omega(\xi)
\end{aligned}
$$

for all $y \in S$. The symbol $\sim$ in the second line should indicate that we assumed that the interchanging of differentiation and integration is possible. Of course, this would have to be proved. Since we prove in the following that the term in (7) is not only the Gâteaux, but also the Fréchet derivative under certain assumptions, we can omit this proof, since the Fréchet derivative is always also the Gâteaux derivative.

Theorem 11 Let $\rho \in \mathrm{C}^{(1)}\left(S^{\mathrm{int}}\right)$. Then, the Fréchet derivative of the operator $\mathcal{S}_{\rho}: \mathrm{C}\left(\mathbb{S}^{2}\right) \rightarrow$ $\mathrm{C}(S)$ is given as

$$
\mathcal{S}_{\rho}^{\prime}[\sigma](\tau)(y)=\int_{\mathbb{S}^{2}} \frac{\rho(\sigma(\xi) \xi)}{|\sigma(\xi) \xi-y|}(\sigma(\xi))^{2} \tau(\xi) \mathrm{d} \omega(\xi)
$$

for all $\sigma, \tau \in \mathrm{C}\left(\mathbb{S}^{2}\right)$ and $y \in S$, assuming there exists $C>0$ such that $|\sigma(\xi) \xi-y|>C$ for all $\xi \in \mathbb{S}^{2}$ and $y \in S$ (i.e., $\Sigma \subseteq S^{\text {int }}$ ).

Proof For the sake of brevity, we define

$$
k(x, y):=\frac{\rho(x)}{|x-y|}|x|^{2}
$$

such that

$$
\mathcal{S}_{\rho}[\sigma](y)=\int_{\mathbb{S}^{2}} \int_{0}^{\sigma(\xi)} k(r \xi, y) \mathrm{d} r \mathrm{~d} \omega(\xi)
$$

Note that

$$
\nabla_{x} k(x, y)=\frac{|x|^{2}}{|x-y|} \nabla_{x} \rho(x)+2 \frac{\rho(x)}{|x-y|} x-\rho(x)|x|^{2} \frac{x-y}{|x-y|^{3}}
$$

and

$$
\left|\nabla_{x} k(x, y)\right| \leq \frac{R_{S^{\text {int }}}^{2}}{C}\|\rho\|_{\mathrm{C}^{(1)}\left(S_{*}^{\text {int }}\right)}+2 \frac{\|\rho\|_{\mathrm{C}\left(S_{*}^{\text {int }}\right)}}{C} R_{S^{\text {int }}}+\|\rho\|_{\mathrm{C}\left(S_{*}^{\text {int }}\right)} R_{S^{\text {int }}}^{2} \frac{1}{C^{2}}<\infty
$$

for all $x \in S_{*}^{\text {int }}$ and $y \in S$, where

$$
S_{*}^{\text {int }}:=\left\{x \in S^{\text {int }}|| x-y \mid>C \text { for all } y \in S\right\}
$$


and $R_{S^{\text {int }}}:=\max _{y \in S}|y|$. Consequently,

$$
\|k(\cdot, y)\|_{\mathrm{C}^{(1)}\left(S_{*}^{\text {int }}\right)}<\infty
$$

and since the right-hand side of (8) does not depend on $y$, we even have

$$
\sup _{y \in S}\|k(\cdot, y)\|_{\mathrm{C}^{(1)}\left(S_{*}^{\text {int }}\right)}<\infty \text {. }
$$

Let $y \in S$ be fixed. Then, if we consider the term that arises from the definition of the Fréchet derivative, we obtain for sufficiently small $\tau \in \mathrm{C}\left(\mathbb{S}^{2}\right)$ that

$$
\begin{aligned}
& \mid \int_{\mathbb{S}^{2}} \int_{0}^{\sigma(\xi)+\tau(\xi)} k(r \xi, y) \mathrm{d} r \mathrm{~d} \omega(\xi)-\int_{\mathbb{S}^{2}} \int_{0}^{\sigma(\xi)} k(r \xi, y) \mathrm{d} r \mathrm{~d} \omega(\xi) \\
& \quad-\int_{\mathbb{S}^{2}} k(\sigma(\xi) \xi, y) \tau(\xi) \mathrm{d} \omega(\xi) \mid \\
& =\left|\int_{\mathbb{S}^{2}} \int_{\sigma(\xi)}^{\sigma(\xi)+\tau(\xi)} k(r \xi, y) \mathrm{d} r \mathrm{~d} \omega(\xi)-\int_{\mathbb{S}^{2}} k(\sigma(\xi) \xi, y) \tau(\xi) \mathrm{d} \omega(\xi)\right| \\
& =\left|\int_{\mathbb{S}^{2}} \int_{\sigma(\xi)}^{\sigma(\xi)+\tau(\xi)} k(r \xi, y) \mathrm{d} r-k(\sigma(\xi) \xi, y) \tau(\xi) \mathrm{d} \omega(\xi)\right| \\
& \leq \int_{\mathbb{S}^{2}}\left|\int_{\sigma(\xi)}^{\sigma(\xi)+\tau(\xi)} k(r \xi, y) \mathrm{d} r-k(\sigma(\xi) \xi, y) \tau(\xi)\right| \mathrm{d} \omega(\xi) \\
& =\int_{\mathbb{S}^{2}}\left|\int_{0}^{\tau(\xi)} k((\sigma(\xi)+r) \xi, y) \mathrm{d} r-k(\sigma(\xi) \xi, y) \tau(\xi)\right| \mathrm{d} \omega(\xi) \\
& =\int_{\mathbb{S}^{2}}|k((\sigma(\xi)+\bar{r}) \xi, y) \tau(\xi)-k(\sigma(\xi) \xi, y) \tau(\xi)| \mathrm{d} \omega(\xi) \\
& =\int_{\mathbb{S}^{2}}|k((\sigma(\xi)+\bar{r}) \xi, y)-k(\sigma(\xi) \xi, y) \| \tau(\xi)| \mathrm{d} \omega(\xi) \\
& =\int_{\mathbb{S}^{2}}\left|\bar{r}\left[\frac{\partial}{\partial r} k((\sigma(\xi)+r) \xi, y)\right]_{r=\hat{r}}\right| \tau(\xi) \mid \mathrm{d} \omega(\xi) \\
& \leq\|k(\cdot, y)\|_{\mathbb{C}^{(1)}\left(S_{*}^{\text {int }}\right)\|\tau\|_{L^{2}\left(\mathbb{S}^{2}\right)}^{2}} \\
& \leq 4 \pi\|k(\cdot, y)\|_{\mathrm{C}^{(1)}\left(S_{*}^{\text {int }}\right)}\|\tau\|_{\left.\mathbb{C}_{(\mathbb{S} 2}\right)}^{2},
\end{aligned}
$$

where we used the triangle inequality in (11), a substitution in (12), the existence of $\bar{r} \in$ $[0, \tau(\xi)]$ such that the equality in (13) holds due to the intermediate value theorem for integrals, and the existence of $\hat{r} \in[0, \bar{r}]$, such that the identity holds due to the intermediate value theorem for differentiation in (14). Furthermore, we employed $\bar{r} \leq|\tau(\xi)|$ and (8) in $(15)$ and the relation between the norms in $\mathrm{L}^{2}\left(\mathbb{S}^{2}\right)$ and $\mathrm{C}\left(\mathbb{S}^{2}\right)$ in (16). Note that we have to require $\tau$ to be sufficiently small such that $|(\sigma(\xi)+\tau(\xi)) \xi-y|>C$ holds, implying a finite $\mathrm{C}^{(1)}\left(S_{*}^{\text {int }}\right)$-norm of $k$.

It follows that

$$
\begin{aligned}
& \sup _{y \in S} \mid \int_{\mathbb{S}^{2}} \int_{0}^{\sigma(\xi)+\tau(\xi)} k(r \xi, y) \mathrm{d} r \mathrm{~d} \omega(\xi)-\int_{\mathbb{S}^{2}} \int_{0}^{\sigma(\xi)} k(r \xi, y) \mathrm{d} r \mathrm{~d} \omega(\xi) \\
& \quad-\int_{\mathbb{S}^{2}} k(\sigma(\xi) \xi, y) \tau(\xi) \mathrm{d} \omega(\xi) \mid \\
& \leq 4 \pi \sup _{y \in S}\|k(\cdot, y)\|_{\mathbb{C}^{(1)}\left(S_{*}^{\text {int })}\right.}\|\tau\|_{\mathbb{C}_{\left(\mathbb{S}^{2}\right)}}^{2}
\end{aligned}
$$


which proves the assertion since the latter term tends to 0 even if it is divided by $\|\tau\|_{C\left(\mathbb{S}^{2}\right)}$ and the term in (10) is exactly the linearization term that arises in the definition of a Fréchet derivative.

Often, one would like to apply Hilbert space techniques in the analysis and numerical solution of inverse problems. Up to now, we have only considered the operator $\mathcal{S}_{\rho}$ as an operator $\mathrm{C}\left(\mathbb{S}^{2}\right) \rightarrow \mathrm{C}(S)$. To consider it as an operator $\mathrm{L}^{2}\left(\mathbb{S}^{2}\right) \rightarrow \mathrm{L}^{2}(S)$, we have to ensure the existence of the integrals in the definition of the operator. Furthermore, we can prove that the image is an $\mathrm{L}^{2}(S)$-function.

Theorem 12 Let $\rho \in \mathrm{L}^{\infty}\left(S^{\text {int }}\right)$ and let $\sigma \in \mathrm{L}^{2}\left(\mathbb{S}^{2}\right)$ such that $|\sigma(\xi) \xi-y|>C$ for almost all $\xi \in \mathbb{S}^{2}$ and some constant $C>0$. Then, we have for almost all $y \in S$ that

$$
\int_{\mathbb{S}^{2}} \int_{0}^{\sigma(\xi)} \frac{\rho(r \xi)}{|r \xi-y|} r^{2} \mathrm{~d} r \mathrm{~d} \omega(\xi)<\infty .
$$

Furthermore, $\mathcal{S}_{\rho}[\sigma] \in \mathrm{L}^{2}(S)$.

Proof Let $k, S_{*}^{\text {int }}$, and $R_{S_{\text {int }}}$ be defined as in the proof of the previous theorem. Then, we have

$$
|k(x, y)|=\frac{|\rho(x)|}{|x-y|}|x|^{2} \leq \frac{\|\rho\|_{L^{\infty}\left(S^{\text {int }}\right)}}{C} R_{S^{\text {int }}}^{2}<\infty
$$

for almost all $(x, y) \in S_{*}^{\text {int }} \times S$ such that

$$
\|k(\cdot, y)\|_{L^{\infty}\left(S_{*}^{\text {int }}\right)}<\infty .
$$

Thus,

$$
\begin{aligned}
\left|\int_{\mathbb{S}^{2}} \int_{0}^{\sigma(\xi)} k(r \xi, y) \mathrm{d} r \mathrm{~d} \omega(\xi)\right| & \leq \int_{\mathbb{S}^{2}}\|k(\cdot, y)\|_{\mathrm{L}^{\infty}\left(S_{*}^{\text {int }}\right)}\left|\int_{0}^{\sigma(\xi)} \mathrm{d} r\right| \mathrm{d} \omega(\xi) \\
& \leq\|k(\cdot, y)\|_{\mathrm{L}^{\infty}\left(S_{*}^{\text {int }}\right)}\|\sigma\|_{\mathrm{L}^{1}\left(\mathbb{S}^{2}\right)} \\
& \leq \sqrt{4 \pi}\|k(\cdot, y)\|_{\mathrm{L}^{\infty}\left(S_{*}^{\text {int }}\right)}\|\sigma\|_{\mathrm{L}^{2}\left(\mathbb{S}^{2}\right)}<\infty
\end{aligned}
$$

for almost all $y \in S$, which proves the first assertion.

Since the penultimate term in (17) does not depend on $y$, we observe that

$$
\begin{aligned}
\int_{S}\left(\mathcal{S}_{\rho}[\sigma](y)\right)^{2} \mathrm{~d} \omega(y) & \leq 4 \pi\|\sigma\|_{\mathrm{L}^{2}\left(\mathbb{S}^{2}\right)}^{2} \int_{S}\left(\|k(\cdot, y)\|_{\mathrm{L}^{\infty}\left(S_{*}^{\text {int }}\right)}\right)^{2} \mathrm{~d} \omega(y) \\
& \leq 4 \pi\|\sigma\|_{\mathrm{L}^{2}\left(\mathbb{S}^{2}\right)}^{2} \int_{S} \frac{\|\rho\|_{\mathrm{L}^{\infty}\left(S^{\mathrm{int}}\right)}^{2}}{C^{2}} R_{S^{\text {int }}}^{4} \mathrm{~d} \omega(y) \\
& =4 \pi\|\sigma\|_{\mathrm{L}^{2}\left(\mathbb{S}^{2}\right)}^{2} \omega(S) \frac{\|\rho\|_{\mathrm{L}^{\infty}\left(S^{\mathrm{int}}\right)}^{2}}{C^{2}} R_{S^{\text {int }}}^{4}<\infty
\end{aligned}
$$

where $\omega(S)$ is the surface measure of the regular surface $S$. This yields $\mathcal{S}_{\rho}[\sigma] \in \mathrm{L}^{2}(S)$.

Using the same technique as in Theorem 11, we can prove the Fréchet differentiability of the operator $\mathcal{S}_{\rho}: \mathrm{L}^{2}\left(\mathbb{S}^{2}\right) \rightarrow \mathrm{L}^{2}(S)$. 
Theorem 13 Let $\rho \in \mathrm{C}^{(1)}\left(S^{\text {int }}\right)$. Then, the Fréchet derivative of the operator $\mathcal{S}_{\rho}: \mathrm{L}^{2}\left(\mathbb{S}^{2}\right) \rightarrow$ $\mathrm{L}^{2}(S)$ is given as

$$
\mathcal{S}_{\rho}^{\prime}[\sigma](\tau)(y)=\int_{\mathbb{S}^{2}} \frac{\rho(\sigma(\xi) \xi)}{|\sigma(\xi) \xi-y|}(\sigma(\xi))^{2} \tau(\xi) \mathrm{d} \omega(\xi)
$$

for all $\sigma, \tau \in \mathrm{L}^{2}\left(\mathbb{S}^{2}\right)$ and $y \in S$, assuming there exists $C>0$ such that $|\sigma(\xi) \xi-y|>C$ for almost all $\xi \in \mathbb{S}^{2}$ and $y \in S$.

Proof Define $k, S_{*}^{\text {int }}$, and $R_{S^{\text {int }}}$ as in the proof of Theorem 11. From (9), we obtain that

$$
\int_{S}\|k(\cdot, y)\|_{\mathrm{C}^{(1)}\left(S_{*}^{\text {int }}\right)}^{2} \mathrm{~d} \omega(y) \leq \omega(S) \sup _{y \in S}\|k(\cdot, y)\|_{\mathrm{C}^{(1)}\left(S_{*}^{\text {int }}\right)}^{2}<\infty .
$$

The inequalities in (10)-(15) are still true such that

$$
\begin{gathered}
\mid \int_{\mathbb{S}^{2}} \int_{0}^{\sigma(\xi)+\tau(\xi)} k(r \xi, y) \mathrm{d} r \mathrm{~d} \omega(\xi)-\int_{\mathbb{S}^{2}} \int_{0}^{\sigma(\xi)} k(r \xi, y) \mathrm{d} r \mathrm{~d} \omega(\xi) \\
-\int_{\mathbb{S}^{2}} k(\sigma(\xi) \xi, y) \tau(\xi) \mathrm{d} \omega(\xi) \mid \\
\leq\|k(\cdot, y)\|_{\mathrm{C}^{(1)}\left(S_{*}^{\text {int }}\right)}\|\tau\|_{\mathrm{L}^{2}\left(\mathbb{S}^{2}\right)}^{2}
\end{gathered}
$$

for almost all $y \in S$. An application of (18) proves the assertion.

\section{Derivation of the algorithm}

We base our considerations on the algorithms of Gauß-Newton type, which are popular methods for the solution of Problem 1, where we restrict ourselves to the case $\mathscr{Y}=\mathbb{R}^{\ell}$, which arises in practice. Examples of these methods are the Levenberg-Marquardt method and the iteratively regularized Gauß-Newton method (cf. Kaltenbacher et al. 2008, Chapter 4 ). The idea of these methods is the iterative minimization of the linearized Tikhonov functional

$$
\left\|g-\mathcal{S}\left[f_{k}\right]-\mathcal{S}^{\prime}\left[f_{k}\right]\left(f_{k+1}-f_{k}\right)\right\|_{\mathbb{R}^{\ell}}^{2}+\lambda_{k+1}\left\|f_{k+1}-f_{k}^{\circ}\right\|_{\mathscr{X}}^{2},
$$

for $f_{k+1} \in \mathscr{X}$, given $g \in \mathbb{R}^{\ell}, f_{k}, f_{k}^{\circ} \in \mathscr{X}$, and $\lambda_{k+1}>0$. Here, $\mathcal{S}^{\prime}[f]: \mathscr{X} \rightarrow \mathbb{R}^{\ell}$ denotes the Fréchet derivative of $\mathcal{S}$ at $f \in \mathscr{X}$.

For a linear operator $\mathcal{T}$, by choosing $f_{k}^{\circ}=0, \lambda_{k+1}=\lambda$ for all $k$, and bearing in mind that $\mathcal{T}^{\prime}[f](h)=\mathcal{T}(h)$, one obtains the same Tikhonov functional that has been used in the derivation of the FMP and the RFMP (see (1)). For nonlinear problems, the term $f_{k}^{\circ}$ in the penalty term takes into account that the zero element in $\mathscr{X}$ plays no special role, in contrast to linear inverse problems, where $\mathcal{T}(0)=0$ if $\mathcal{T}$ is linear.

The already mentioned Gauß-Newton methods solve the minimization of the functional in (19) by solving the corresponding (regularized) normal equation. This, consequently, yields the iterative scheme

$$
\left(\mathcal{S}^{\prime}\left[f_{k}\right]^{*} \mathcal{S}^{\prime}\left[f_{k}\right]+\lambda \mathcal{I}\right)\left(f_{k+1}-f_{k}\right)=\mathcal{S}^{\prime}\left[f_{k}\right]^{*}\left(g-\mathcal{S}\left[f_{k}\right]\right)+\lambda_{k+1}\left(f_{k}^{\circ}-f_{k}\right) .
$$

Here, the Levenberg-Marquardt method and the iteratively regularized Gauß-Newton method correspond to $f_{k}^{\circ}=f_{k}$ and $f_{k}^{\circ}=f_{0}$, respectively. 
We will now use the idea of iteratively minimizing the functional in (19) to obtain a greedy algorithm for nonlinear inverse problems. In analogy to the RFMP, we choose a dictionary $\mathscr{D} \subseteq \mathscr{X}$, a fixed regularization parameter $\lambda>0$, and an initial approximation $f_{0} \in \mathscr{X}$. We then iteratively define a sequence $\left(f_{k}\right)_{k \in \mathbb{N}_{0}}$ of approximations to the solution $f^{*}$ by

$$
f_{k+1}:=f_{k}+\alpha_{k+1} d_{k+1} .
$$

We will now determine how $\alpha_{k+1} \in \mathbb{R}$ and $d_{k+1} \in \mathscr{D}$ have to be chosen to minimize the linearized Tikhonov functional

$$
\widetilde{\mathcal{A}}_{\lambda}\left[g, f_{k}, f_{k}^{\circ}, d, \alpha\right]=\left\|g-\mathcal{S}\left[f_{k}\right]-\alpha \mathcal{S}^{\prime}\left[f_{k}\right](d)\right\|_{\mathbb{R}^{\ell}}^{2}+\lambda\left\|\left(f_{k}-f_{k}^{\circ}\right)+\alpha d\right\|_{\mathscr{X}}^{2},
$$

for given $g \in \mathbb{R}^{\ell}, \lambda>0$, and $f_{k}, f_{k}^{\circ} \in \mathscr{X}$. Using the technique of the derivation of the RFMP from Fischer (2011); Fischer and Michel (2012); Michel (2015), we first observe that

$$
\begin{aligned}
\widetilde{\mathcal{A}}_{\lambda}\left[g, f_{k}, f_{k}^{\circ}, d, \alpha\right]= & \left\|r_{k}\right\|_{\mathscr{Y}}^{2}-2 \alpha\left\langle r_{k}, \mathcal{S}^{\prime}\left[f_{k}\right](d)\right\rangle_{\mathscr{Y}}+\alpha^{2}\left\|\mathcal{S}^{\prime}\left[f_{k}\right](d)\right\|_{\mathscr{Y}}^{2} \\
& +\lambda\left(\left\|f_{k}-f_{k}^{\circ}\right\|_{\mathscr{X}}^{2}+2 \alpha\left\langle f_{k}-f_{k}^{\circ}, d\right\rangle_{\mathscr{X}}+\alpha^{2}\|d\|_{\mathscr{X}}^{2}\right) \\
= & \left(\left\|r_{k}\right\|_{\mathscr{Y}}^{2}+\lambda\left\|f_{k}-f_{k}^{\circ}\right\|_{\mathscr{X}}^{2}\right) \\
& -2 \alpha\left(\left\langle r_{k}, \mathcal{S}^{\prime}\left[f_{k}\right](d)\right\rangle_{\mathscr{Y}}-\lambda\left\langle f_{k}-f_{k}^{\circ}, d\right\rangle_{\mathscr{X}}\right) \\
& +\alpha^{2}\left(\left\|\mathcal{S}^{\prime}\left[f_{k}\right](d)\right\|_{\mathscr{Y}}^{2}+\lambda\|d\|_{\mathscr{X}}^{2}\right)
\end{aligned}
$$

where $r_{k}:=g-\mathcal{S}\left[f_{k}\right]$. For fixed $d \in \mathscr{D}$, a necessary condition for the minimization of $\widetilde{\mathcal{A}}_{\lambda}$ is

$$
\begin{aligned}
0= & \frac{\partial}{\partial \alpha} \widetilde{\mathcal{A}}_{\lambda}\left[g, f_{k}, f_{k}^{\circ}, d, \alpha\right] \\
=- & 2\left(\left\langle r_{k}, \mathcal{S}^{\prime}\left[f_{k}\right](d)\right\rangle_{\mathscr{Y}}-\lambda\left\langle f_{k}-f_{k}^{\circ}, d\right\rangle_{\mathscr{X}}\right) \\
& +2 \alpha\left(\left\|\mathcal{S}^{\prime}\left[f_{k}\right](d)\right\|_{\mathscr{Y}}^{2}+\lambda\|d\|_{\mathscr{X}}^{2}\right),
\end{aligned}
$$

which, for the minimizer $\alpha=\alpha_{k+1}$, is equivalent to

$$
\alpha_{k+1}=\frac{\left\langle r_{k}, \mathcal{S}^{\prime}\left[f_{k}\right](d)\right\rangle_{\mathscr{Y}}-\lambda\left\langle f_{k}-f_{k}^{\circ}, d\right\rangle_{\mathscr{X}}}{\left\|\mathcal{S}^{\prime}\left[f_{k}\right](d)\right\|_{\mathscr{Y}}^{2}+\lambda\|d\|_{\mathscr{X}}^{2}} .
$$

Note that $\widetilde{\mathcal{A}}_{\lambda}$ is convex with respect to $\alpha$, since it can be seen in (20) that it corresponds to a quadratic polynomial with a positive leading coefficient. Thus, the condition in (21) is not only necessary but also sufficient for the minimization of $\widetilde{\mathcal{A}}_{\lambda}$.

Inserting (22) into (20) yields

$$
\begin{aligned}
\widetilde{\mathcal{A}}_{\lambda}\left[g, f_{k}, f_{k}^{\circ}, d, \alpha_{k+1}\right]= & \left.\left\|r_{k}\right\|_{\mathscr{Y}}^{2}+\lambda\left\|f_{k}-f_{k}^{\circ}\right\|_{\mathscr{X}}^{2}\right) \\
& -2 \frac{\left(\left\langle r_{k}, \mathcal{S}^{\prime}\left[f_{k}\right](d)\right\rangle_{\mathscr{Y}}-\lambda\left\langle f_{k}-f_{k}^{\circ}, d\right\rangle_{\mathscr{X}}\right)^{2}}{\left\|\mathcal{S}^{\prime}\left[f_{k}\right](d)\right\|_{\mathscr{Y}}^{2}+\lambda\|d\|_{\mathscr{X}}^{2}} \\
& +\frac{\left(\left\langle r_{k}, \mathcal{S}^{\prime}\left[f_{k}\right](d)\right\rangle_{\mathscr{Y}}-\lambda\left\langle f_{k}-f_{k}^{\circ}, d\right\rangle_{\mathscr{X}}\right)^{2}}{\left\|\mathcal{S}^{\prime}\left[f_{k}\right](d)\right\|_{\mathscr{Y}}^{2}+\lambda\|d\|_{\mathscr{X}}^{2}} \\
= & \left(\left\|r_{k}\right\|_{\mathscr{Y}}^{2}+\lambda\left\|f_{k}-f_{k}^{\circ}\right\|_{\mathscr{X}}^{2}\right) \\
& -\frac{\left(\left\langle r_{k}, \mathcal{S}^{\prime}\left[f_{k}\right](d)\right\rangle_{\mathscr{Y}}-\lambda\left\langle f_{k}-f_{k}^{\circ}, d\right\rangle_{\mathscr{X}}\right)^{2}}{\left\|\mathcal{S}^{\prime}\left[f_{k}\right](d)\right\|_{\mathscr{Y}}^{2}+\lambda\|d\|_{\mathscr{X}}^{2}} .
\end{aligned}
$$


We observe that the first term does not depend on $d$. Thus, the pair $\left(\alpha_{k+1}, d_{k+1}\right) \in \mathbb{R} \times \mathscr{D}$ is a minimizer of $\widetilde{\mathcal{A}}_{\lambda}\left[g, f_{k}, f_{k}^{\circ}, \cdot, \cdot\right]$ if and only if

$$
\begin{aligned}
& d_{k+1}=\underset{d \in \mathscr{D}}{\operatorname{argmax}} \frac{\left(\left\langle r_{k}, \mathcal{S}^{\prime}\left[f_{k}\right](d)\right\rangle_{\mathscr{Y}}-\lambda\left\langle f_{k}-f_{k}^{\circ}, d\right\rangle_{\mathscr{X}}\right)^{2}}{\left\|\mathcal{S}^{\prime}\left[f_{k}\right](d)\right\|_{\mathscr{Y}}^{2}+\lambda\|d\|_{\mathscr{X}}^{2}}, \\
& \alpha_{k+1}=\frac{\left\langle r_{k}, \mathcal{S}^{\prime}\left[f_{k}\right]\left(d_{k+1}\right)\right\rangle_{\mathscr{Y}}-\lambda\left\langle f_{k}-f_{k}^{\circ}, d_{k+1}\right\rangle_{\mathscr{X}}}{\left\|\mathcal{S}^{\prime}\left[f_{k}\right]\left(d_{k+1}\right)\right\|_{\mathscr{Y}}^{2}+\lambda\left\|d_{k+1}\right\|_{\mathscr{X}}^{2}}
\end{aligned}
$$

holds if we assume that a maximizer exists in the first equation. These two identities will be the key ingredient of the following algorithm.

Algorithm 14 (RFMP for Nonlinear Problems, RFMP_NL) Let $\mathcal{S}: \mathscr{X} \rightarrow \mathbb{R}^{\ell}$ and $g \in$ $\mathbb{R}^{d}$ be given as in Problem 1. Choose a dictionary $\mathscr{D} \subseteq \mathscr{X} \backslash\{0\}$, an initial approximation $f_{0} \in \mathscr{X}$, and a regularization parameter $\lambda>0$. Furthermore, specify the type of regularization by choosing the sequence $f_{k}^{\circ} \in \mathscr{X}$, for example, as one of the options stated above.

1. Set $k:=0$, define the residual $r_{0}:=g-\mathcal{S}\left[f_{0}\right]$ and choose a stopping criterion.

2. Find

$$
d_{k+1}=\underset{d \in \mathscr{D}}{\operatorname{argmax}} \frac{\left(\left\langle r_{k}, \mathcal{S}^{\prime}\left[f_{k}\right](d)\right\rangle_{\mathscr{Y}}-\lambda\left\langle f_{k}-f_{k}^{\circ}, d\right\rangle_{\mathscr{X}}\right)^{2}}{\left\|\mathcal{S}^{\prime}\left[f_{k}\right](d)\right\|_{\mathscr{Y}}^{2}+\lambda\|d\|_{\mathscr{X}}^{2}}
$$

and set

$$
\alpha_{k+1}:=\frac{\left\langle r_{k}, \mathcal{S}^{\prime}\left[f_{k}\right]\left(d_{k+1}\right)\right\rangle_{\mathscr{Y}}-\lambda\left\langle f_{k}-f_{k}^{\circ}, d_{k+1}\right\rangle_{\mathscr{X}}}{\left\|\mathcal{S}^{\prime}\left[f_{k}\right]\left(d_{k+1}\right)\right\|_{\mathscr{Y}}^{2}+\lambda\left\|d_{k+1}\right\|_{\mathscr{X}}^{2}},
$$

as well as $f_{k+1}:=f_{k}+\alpha_{k+1} d_{k+1}$ and $r_{k+1}:=g-\mathcal{S}\left[f_{k+1}\right]$.

3. If the stopping criterion is satisfied, then $f_{k+1}$ is the output. Otherwise, increase $k$ by 1 and return to step 2.

\section{Comparison to other methods}

Most methods for nonlinear inverse problems are iterative (for an overview, see, for example, the book Kaltenbacher et al. 2008). Examples are the already mentioned Gauß-Newton methods, where a linearized Tikhonov functional (therefore Newton) is minimized by solving a normal equation (therefore Gauß). The linearized Tikhonov functional itself is obtained by applying a Tikhonov regularization to the linearized equation that would be solved by a pure Newton method. Representatives of this category of methods are the LevenbergMarquardt method (see Levenberg 1944; Marquardt 1963) and the iteratively regularized Gauß-Newton method (see Bakushinsky 1992). A substantially different classical category of methods for nonlinear inverse problems are gradient-type methods, in particular, the Landweber method, which can be applied to linear (see Landweber 1951) and nonlinear inverse problems (see Hanke et al. 1995). Furthermore, (direct) Tikhonov regularization methods (see Tikhonov and Glasko 1965), multilevel methods (see Kaltenbacher et al. 2008, Chapter 5), and sequential subspace optimization methods (see Wald and Schuster 2017) have been developed for nonlinear inverse problems. Especially, we want to mention level set methods (see, for example, the survey by Burger and Osher 2005), since these are methods that are often used for problems like the nonlinear inverse gravimetric problem, where a domain is the unknown. Although the latter possess several advantageous properties, for example, one is able to recover domains that are not star-shaped or even unconnected, one 
does not directly get an explicit representation of the surface of the unknown domain (although one can try to obtain such a representation in a post-processing step). In contrast, we directly obtain such a representation in spherical coordinates, which is desirable from the geophysical perspective. Furthermore, the assumptions that the Earth's interior (and also the part of the Earth that is inside a boundary layer like the Mohorovičić discontinuity) is connected or even star-shaped is a good model of the reality.

In the following, we want to compare the newly developed RFMP_NL with existing methods for nonlinear problems from the algorithmic point of view. First, there are several similarities between all of the methods. The RFMP_NL is an iterative method, like most of the established methods. Even if these are not iterative by themselves, like Tikhonov regularization, often iterative optimization algorithms have to be used inside these algorithms. As stated above, the derivation of the RFMP_NL was actually motivated by Gauß-Newton methods, which shows the clear similarity to these methods.

Secondly, there are also several differences to other methods, both advantages and disadvantages. A disadvantage of the RFMP_NL is the fact that we currently do not have an accurate theoretical analysis of the method, that is, no convergence or regularization result. These results have already been very elaborate to obtain in the case of greedy algorithms (RFMP, ROFMP, RWFMP) for linear inverse problems (see the considerations in Fischer 2011; Kontak 2018; Kontak and Michel 2018; Michel 2015; Michel and Orzlowski 2017; Michel and Telschow 2016; Telschow 2014), and we expect it to be much more difficult in the nonlinear case. Moreover, since the dictionary does not need to form a basis of the underlying Hilbert space, the linear combination of the approximation that is provided by the RFMP_NL may not be optimal, since the approximation has no unique representation with respect to the dictionary. Nevertheless, the RFMP_NL also has several advantages in comparison to the existing methods. Most of these other methods are given in a pure, infinite-dimensional, Hilbert (or Banach) space formulation. Naturally, it is not possible to implement these methods directly on a computer. Thus, one has to choose a specific basis system in order to implement the method. It is necessary that this system is a basis to ensure the regularity of the arising linear systems, for example, in the Levenberg-Marquardt method and using ansatz functions for the solution that are linearly dependent may lead to singular matrices. In contrast, the RFMP_NL can handle very diverse types of basis functions (for example, global and localized functions) and will choose those functions that are best adapted to the structure of the solution. This is especially important in those applications, for example, in geophysics, where global structures like the Earth's ellipticity must be distinguished from local structures like mountains. Furthermore, there even is no need at all to solve linear systems, like in most of the other methods. Thus, one does not need to care about the condition of arising matrices, which may require a stabilization, and there is also no need to apply iterative solvers for the arising linear systems, which is often the most efficient way when implementing the other methods. Furthermore, an additional advantage of the RFMP_NL is that there is no need to know the adjoint operator of the Fréchet derivative. The operator itself even does not need to be Fréchet differentiable. Since the linearization of operators (like in the linearized Tikhonov functional) is also possible using the Gâteaux derivative (see, for example, Cea 1978, Section 1.2), Gâteaux differentiability would actually be enough in this context. 


\section{Application to the nonlinear inverse gravimetric problem}

Here, we will apply the RFMP_NL, which was derived in Section 4, to the nonlinear inverse gravimetric problem, which was presented in Section 3.

First, we will give some details about the implementation of the algorithm. Then, we will present numerical results for two synthetic scenarios, where we use a variation of dictionaries and prescribed solutions.

6.1 Details of the implementation

The algorithm was implemented in the C Programming Language (see Kerningham and Ritchie 1988) using the GNU Scientific Library (see Galassi et al. 2009), and a parallelization with OpenMP (see Dagum and Menon 1998; OpenMP Architecture Review Board 2013).

Besides the implementation of the algorithm itself, which we will discuss later in this section, we need an implementation of the nonlinear operator that is associated to the nonlinear inverse gravimetric problem as well as its derivative.

Remember that the operator $\mathcal{S}_{\rho}$ of the nonlinear inverse gravimetric problem is given as

$$
\mathcal{S}_{\rho}[\sigma](y)=\int_{\mathbb{S}^{2}} \int_{0}^{\sigma(\xi)} \frac{\rho(r \xi)}{|r \xi-y|} r^{2} \mathrm{~d} r \mathrm{~d} \omega(\xi), \quad y \in S,
$$

and its Fréchet and Gâteaux derivatives possess the form

$$
\mathcal{S}_{\rho}^{\prime}[\sigma](\tau)(y)=\int_{\mathbb{S}^{2}} \frac{\rho(\sigma(\xi) \xi)}{|\sigma(\xi) \xi-y|}(\sigma(\xi))^{2} \tau(\xi) \mathrm{d} \omega(\xi), \quad y \in S .
$$

We will restrict ourselves to constant density $\rho \equiv 1$ in all of the numerical examples. For the sake of readability, as in the proof of Theorem 11, we define the function $k: S^{\text {int }} \times S \rightarrow \mathbb{R}$,

$$
k(x, y):=\frac{1}{|x-y|}|x|^{2}
$$

such that

$$
\begin{aligned}
\mathcal{S}_{\rho}[\sigma](y) & =\int_{\mathbb{S}^{2}} \int_{0}^{\sigma(\xi)} k(r \xi, y) \mathrm{d} r \mathrm{~d} \omega(\xi), \\
\mathcal{S}_{\rho}^{\prime}[\sigma](\tau)(y) & =\int_{\mathbb{S}^{2}} k(\sigma(\xi) \xi, y) \tau(\xi) \mathrm{d} \omega(\xi) .
\end{aligned}
$$

We first deal with a numerical integration method on $\mathbb{S}^{2}$, since it is needed in both (23) and (24). The method, which we will use, was developed in Driscoll and Healy (1994) and is based on the following point grid.

Definition 15 (cf. Michel 2013, Theorem 7.33) Let $m \in \mathbb{N}$. Let the points $\eta_{p, q}=\eta\left(\varphi_{q}, t_{p}\right) \in$ $\mathbb{S}^{2}$ be defined by the polar coordinates

$$
\begin{aligned}
\varphi_{q} & =\frac{2 \pi q}{m+1}, & q & =0, \ldots, m, \\
t_{p} & =\cos \left(\frac{\pi p}{m+1}\right), & p & =0, \ldots, m .
\end{aligned}
$$

Then, $\left\{\eta_{p, q} \mid p, q=0, \ldots, m\right\}$ is called the Driscoll-Healy grid. 
For spherical harmonics up to degree $m$, there exists an exact integration formula using the Driscoll-Healy grid.

Theorem 16 (cf. Michel 2013, Theorem 7.33) Let $f \in \operatorname{Harm}_{0 . \ldots m}\left(\mathbb{S}^{2}\right)$ for an odd number $m \in \mathbb{N}$ and let $\left\{\eta_{p, q} \mid p, q=0, \ldots, m\right\}$ be the Driscoll-Healy grid. Let the integration weights $a_{0}, \ldots, a_{m} \in \mathbb{R}$ be defined as

$$
a_{p}:=\frac{4}{m+1} \sin \left(\frac{\pi p}{m+1}\right) \sum_{s=0}^{(m+1) / 2-1} \frac{1}{2 s+1} \sin \left((2 s+1) \frac{\pi p}{m+1}\right)
$$

for $p=0, \ldots, m$.

Then,

$$
\int_{\mathbb{S}^{2}} f(\eta) \mathrm{d} \omega(\eta)=\frac{2 \pi}{m+1} \sum_{p=0}^{m} a_{p} \sum_{q=0}^{m} F\left(\eta_{p, q}\right) .
$$

For all of the arising integrals over the sphere, we use exactly this method with parameter $m=99$. It remains to choose an integration method for the radial integral in (23). We apply a simple partitioned trapezoidal rule with 100 points.

An alternative for the numerical calculation of the integral in (23) is to transform the integral to the ball $\mathbb{B}_{1}$ such that

$$
\begin{aligned}
\int_{\mathbb{S}^{2}} \int_{0}^{\sigma(\xi)} k(r \xi, y) \mathrm{d} r \mathrm{~d} \omega(\xi) & =\int_{\mathbb{S}^{2}} \int_{0}^{1} k(t \sigma(\xi) \xi, y) \sigma(\xi) \mathrm{d} t \mathrm{~d} \omega(\xi) \\
& =\int_{\mathbb{B}_{1}} k\left(|x| \sigma\left(\frac{x}{|x|}\right) \frac{x}{|x|}, y\right) \sigma\left(\frac{x}{|x|}\right) \frac{1}{|x|^{2}} \mathrm{~d} x
\end{aligned}
$$

and apply an integration method for the ball, as for example derived by Amna and Michel (2017), but for the simplicity of the implementation, we do not pursue this approach.

The general procedure for the presented numerical experiments will be as follows.

1. Prescribe a solution $\sigma_{\mathrm{sol}}: \mathbb{S}^{2} \rightarrow(0, \infty)$ and use numerical integration to compute synthetic data $g_{j}:=\mathcal{S}_{1}\left[\sigma_{\text {sol }}\right]\left(y_{j}\right), j=1, \ldots, J$, where $\left(y_{j}\right)_{j=1, \ldots, J} \subseteq S$ are points on a sphere with radius $R, S:=\mathbb{S}_{R}^{2}:=\left\{x \in \mathbb{R}^{3}|| x \mid=R\right\}$. Ensure that $R>\sup _{\xi \in \mathbb{S}^{2}} \sigma_{\text {sol }}(\xi)$.

2. Apply $1 \%$ of noise to $g_{j}$.

3. Choose a function space $\mathscr{X}\left(\mathbb{S}^{2}\right) \subseteq \mathrm{L}^{2}\left(\mathbb{S}^{2}\right)$ and a dictionary $\mathscr{D} \subseteq \mathscr{X}\left(\mathbb{S}^{2}\right) \backslash\{0\}$.

4. Define a set $\left(\lambda_{s}\right)_{s=1, \ldots, S}$ of regularization parameters and define the sequence $\left(f_{k}^{\circ}\right)_{k}$ of functions, which determines the type of regularization.

5. For each regularization parameter, run the RFMP_NL using the noisy data and the predefined dictionary.

6. Choose the regularization parameter, which yields the lowest approximation error.

Note that, in practice, one should ensure that the condition $R>\sup _{\xi \in \mathbb{S}^{2}} \sigma_{\text {sol }}(\xi)$ is also true for $R-\varepsilon$ instead of $R$ for some sufficiently large $\varepsilon>0$, since noisy data and inaccuracies in the calculation of the approximate solution could cause larger values of $\sigma$ in comparison to the exact solution. In our numerical examples, the sphere where the data are given is, indeed, at a sufficiently high altitude which is larger than the maximum of the recovered solution.

In general, we will look at two different scenarios. The first one will use a contrived solution with purely global structures and a dictionary of spherical harmonics for a specific combination of the regularization term and the function space. The second one will combine 


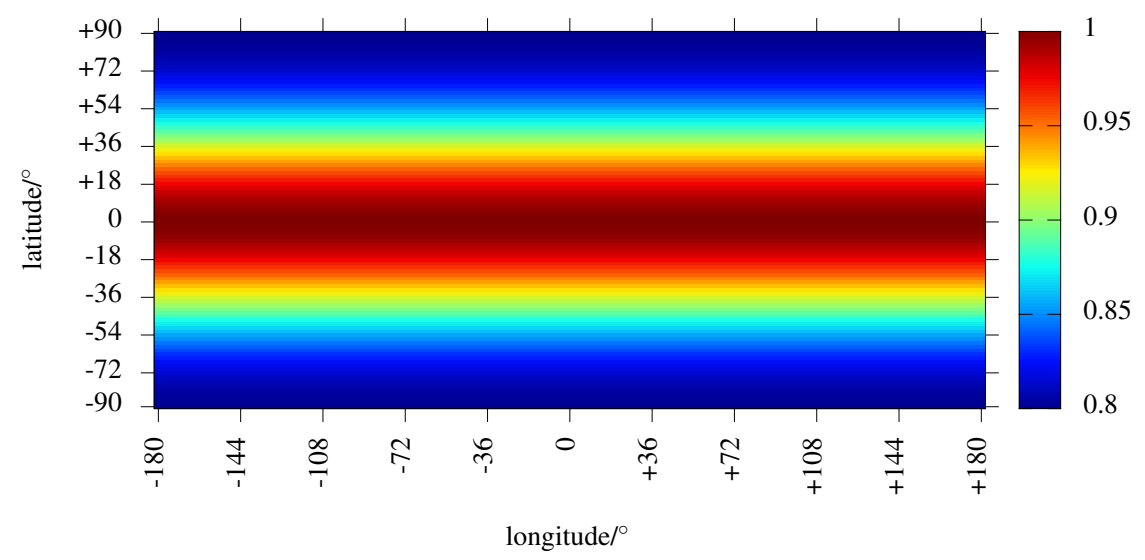

Figure 3: Prescribed solution in Example 1: describes an ellipsoid of revolution with aspect ratio 0.8

both global and local features in the prescribed solution and correspondingly, the dictionary will consist of spherical harmonics and Abel-Poisson kernels. For the second scenario, we will compare the different alternatives for the regularization term and the function space. For the combination that yields the lowest approximation error we will present concrete results for the two different prescribed solutions.

\subsection{Example 1: ellipsoid of revolution}

We prescribe the solution

$$
f(\xi):=\sigma_{\mathrm{sol}}(\xi):=\frac{1}{\sqrt{\xi_{1}^{2}+\xi_{2}^{2}+\left(\frac{\xi_{3}}{0.8}\right)^{2}}},
$$

such that it can easily be seen that

$$
\Sigma^{\text {int }}=\left\{r \xi \in \mathbb{R}^{3} \mid \xi \in \mathbb{S}^{2}, r<\sigma(\xi)\right\}
$$

represents an ellipsoid of revolution with aspect ratio 0.8. The solution is depicted in Figure 3 in spherical coordinates. We evaluated the gravitational potential in 10000 points on a Driscoll-Healy grid with $m=99$ on a sphere of radius 1.1 .

The prescribed solution consists only of global structures. Consequently, we chose a dictionary that consists of global functions, namely, spherical harmonics, only. The dictionary

$$
\mathscr{D}:=\left\{Y_{n, j} \mid n=0, \ldots, 25 ; j=-n, \ldots, n\right\}
$$

consists of all spherical harmonics up to degree and order 25 such that $\# \mathscr{D}=676$. We chose the function space $\mathscr{X}\left(\mathbb{S}^{2}\right)=\mathrm{L}^{2}\left(\mathbb{S}^{2}\right)$ and set $f_{k}^{\circ}:=f_{0}$ for $k \in \mathbb{N}_{0}$ such that the regularization term corresponds to the term that is also used in the iteratively regularized GaußNewton method. As the initial approximation, we chose $f_{0} \equiv 0.8$, corresponding to the 
sphere with radius 0.8. We performed 200 iterations of the RFMP_NL. As already mentioned, we chose the regularization parameter by executing the algorithm for a sequence of regularization parameters and obtained $\lambda=1.585 \times 10^{-2}$ as the regularization parameter, which minimizes the approximation error. The development of the relative residual, that is, $\left\|g-\mathcal{S}\left[f_{k}\right]\right\|_{\mathscr{Y}} /\|g\|_{\mathscr{Y}}$, and the relative approximation error, that is, $\left\|f-f_{k}\right\|_{\mathscr{X}} /\|f\|_{\mathscr{X}}$, during the iteration can be found in Figure 4A. The approximation after 200 iterations is depicted in Figure 4B. Moreover, the pointwise difference of the approximation after 200 iterations and the solution is shown in Figure 4C.

In the analysis of Figure 4A, we first consider the residual. We observe that the relative residual drops rapidly below $1 \%$ in the first few iterations. Since we used a noise level of $1 \%$ in the data space, this is what we would expect. This also shows that the algorithm works as it should, since (ignoring the regularization) it was derived as a minimization algorithm for the (linearized) residual. Considering the relative approximation error, we observe that the final value after 200 iterations is approximately $1.4 \%$. Due to the ill-posedness and the nonlinearity of the inverse problem, it is not surprising that the approximation error is larger than the residual and the noise level. Indeed, a factor of 1.4 between the approximation error and the noise level is a good result for an algorithm for ill-posed inverse problems. Unfortunately, we also observe that the error is even lower in several of the earlier iterations. It would, consequently, be even more efficient to stop the algorithm, when the error is minimal. Since, in general, we do not know the solution, this cannot be achieved. Looking at the results that we obtained by the RFMP_NL for all of the other regularization parameters (not shown here), we can say that for these parameters the difference between the minimal error and the error after 200 iterations is even larger such that, currently, the results presented in Figure 4 are the best we could achieve. Of course, one could also think about stopping the iteration earlier, for example, using a discrepancy principle, which is a common procedure for iterative regularization methods. From methods like the Levenberg-Marquardt algorithm, it is known that one would have to choose a different regularization parameter in every iteration, which is even more difficult. This is why we postpone this subject to our future research.

The approximation itself and the approximation error as a function in spherical coordinates are shown in Figures 4B and 4C, respectively. In addition to the plot of the approximation error in spherical coordinates in Figure 4C, we also provide a three-dimensional plot on the sphere in Figure 5 to account for the misperception of structures around the poles that might arise due to the cartographic projection. It can be seen that the error is very small everywhere on the sphere. Its absolute value is nowhere larger than 0.01 , where the surface of the body of mass is between 0.8 and 1 units away from the center. We find that there are several small artefacts in the approximation error distributed over the whole sphere. From the application of the RFMP (and other regularization methods) to linear inverse problems, we know that such artefacts normally arise if the regularization parameter is chosen too low. As already said, we observed larger approximation errors for larger values of the regularization parameter such that we do not believe that the artefacts arise from under-regularization.

Nevertheless, we can say that, for this example, the approximations generated by the RFMP_NL after 200 iterations are very good, since the relative approximation error is only $1.4 \%$ for a noise level of $1 \%$. 


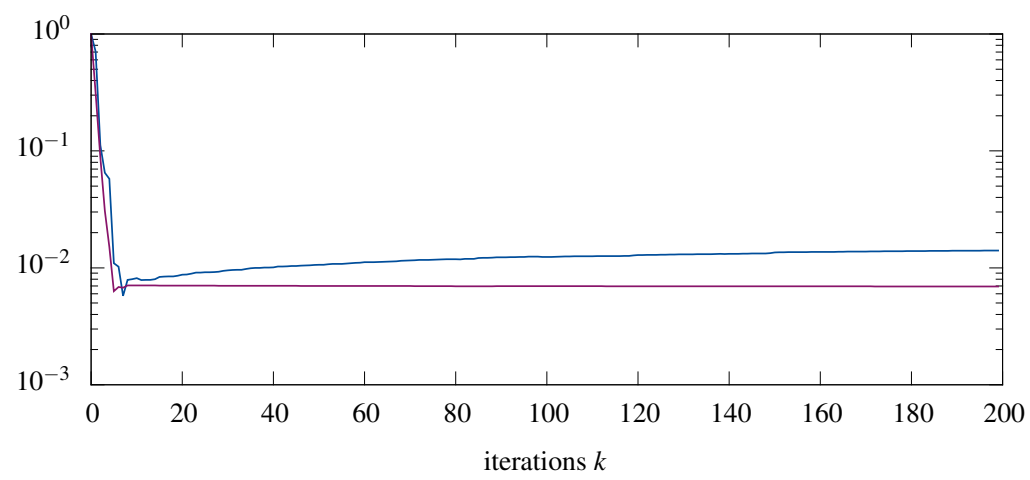

(a) Development of the relative residual (purple) and the relative approximation error (blue) during the iteration of the RFMP_NL.

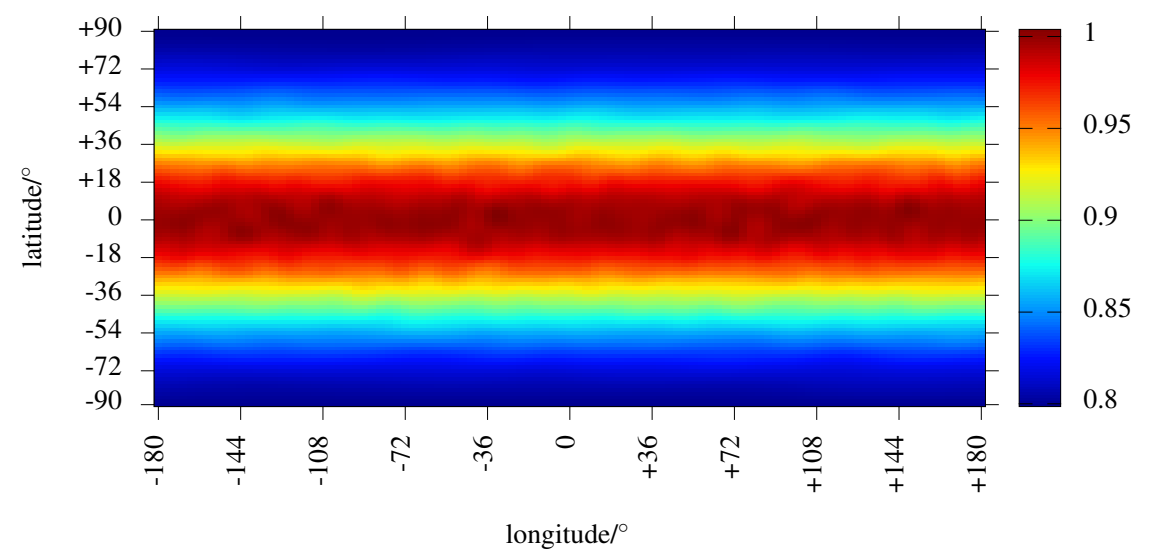

(b) Approximation generated by the RFMP_NL.

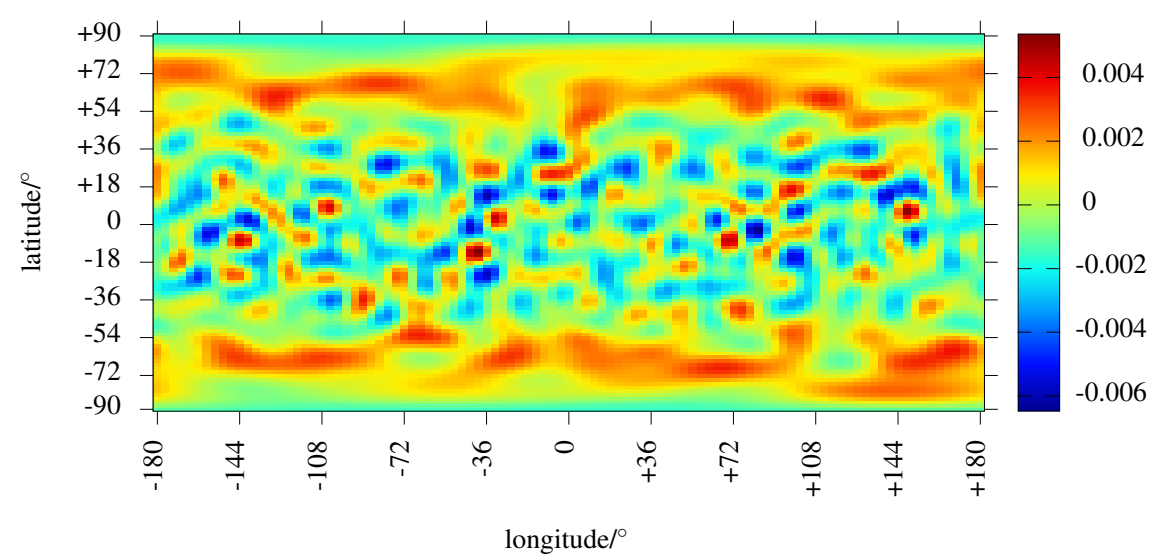

(c) Difference of the solution and the approximation generated by the RFMP_NL.

Figure 4: Results from the application of the RFMP_NL in Example 1. 


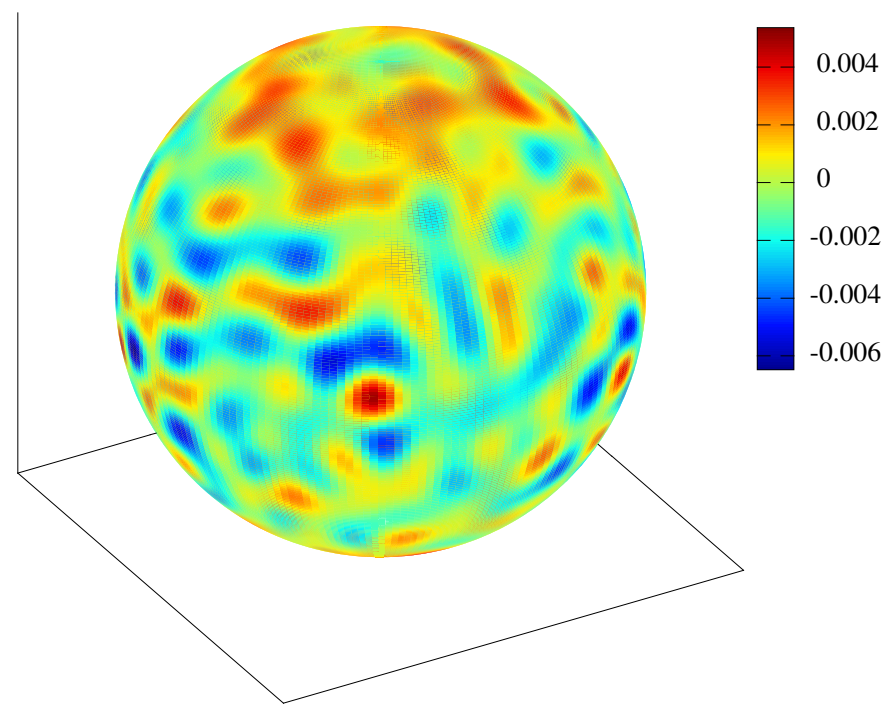

Figure 5: Three-dimensional plot of the approximation error after 200 iterations of the RFMP_NL in Example 1 as a function on the sphere.

\subsection{Example 2: ellipsoid and added Abel-Poisson kernels}

In the introduction, we stressed that the RFMP for linear inverse problems proved to be able to combine different types of basis functions. Therefore, in this section we will discuss an example, which will show that this is also true in practice for the RFMP_NL.

In this case, we will first compare different possibilities for the chosen function spaces and the regularization term. Afterwards, we will consider the numerical results of the combination of a function space and a regularization term that yielded the smallest error.

We chose a dictionary $\mathscr{D}$ that consists of spherical harmonics up to degree 9 and AbelPoisson kernels with parameter $h=0.7$, which are centered on a Driscoll-Healy grid with parameter $m=25$, yielding 100 spherical harmonics and 652 Abel-Poisson kernels to obtain $\# \mathscr{D}=752$ in total. The prescribed solution consisted of a sum of the function used in the first example corresponding to an ellipsoid of revolution and two Abel-Poisson kernels with parameter $h=0.7$, which are centered at $41^{\circ} \mathrm{N}, 96^{\circ} \mathrm{W}$ and $41^{\circ} \mathrm{S}, 96^{\circ} \mathrm{E}$. The solution is depicted in Figure 6.

To obtain data for this second synthetic example, we evaluated the gravitational potential at 10000 points on a Driscoll-Healy grid on the sphere with radius 1.2.

In Table 1, we gather the approximation errors for two different types of regularization terms and three different function spaces $\mathscr{X}\left(\mathbb{S}^{2}\right)$. For every combination, we executed 200 iterations of the RFMP_NL with several regularization parameters. The values in the table are the lowest approximation errors that we obtained among all of the regularization parameters. As already mentioned before, choosing $f_{k}^{\circ}=f_{0}$ in the RFMP_NL is analogous to the iteratively regularized Gauß-Newton method, and $f_{k}^{\circ}=f_{k}$ is analogous to the Levenberg-Marquardt method. This is the reason why we compare these two choices for the 


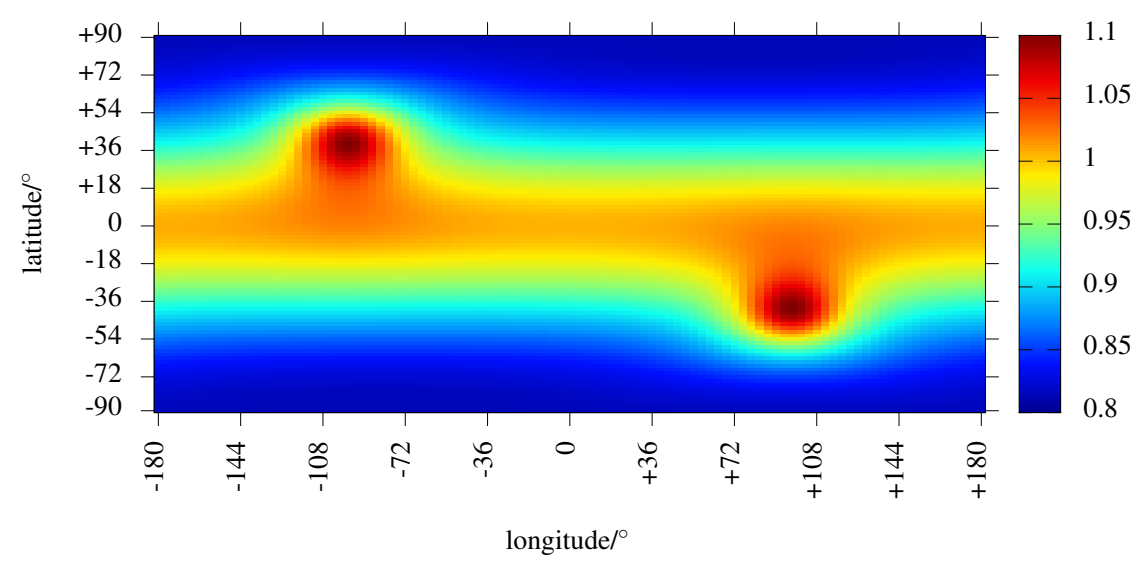

Figure 6: Prescribed solution in Example 2: the sum of an ellipsoid of revolution and two Abel-Poisson kernels.

Table 1: Approximation errors for different choices of the regularization term and the function spaces in Example 2. The minimum approximation error is set in bold font.

\begin{tabular}{lll}
\hline Function space & $f_{k}^{\circ}=f_{0}$ & $f_{k}^{\circ}=f_{k}$ \\
\hline $\mathrm{L}^{2}\left(\mathbb{S}^{2}\right)$ & $3.10 \%$ & $3.20 \%$ \\
$\mathrm{H}^{1}\left(\mathbb{S}^{2}\right)$ & $3.26 \%$ & $3.23 \%$ \\
$\mathrm{H}^{2}\left(\mathbb{S}^{2}\right)$ & $3.30 \%$ & $3.22 \%$ \\
\hline
\end{tabular}

regularization term. Apart from the space $\mathrm{L}^{2}\left(\mathbb{S}^{2}\right)$, we also considered the inverse problem in the Sobolev spaces $\mathrm{H}^{1}\left(\mathbb{S}^{2}\right)$ and $\mathrm{H}^{2}\left(\mathbb{S}^{2}\right)$ (for a definition see, for example, Freeden et al. 1998, Section 5.1).

We observe that the combination of $f_{k}^{\circ}=f_{0}$ and the space $\mathrm{L}^{2}\left(\mathbb{S}^{2}\right)$ yields the best results, although all of the other results have the same order of magnitude. We, consequently, stick to this combination in the further analysis of the results. This also has the advantage that $\mathrm{L}^{2}\left(\mathbb{S}^{2}\right)$-norms of the functions in the dictionary, in general, can be more easily computed compared to the Sobolev norms. There, one has to compute a truncated Legendre series, which is much more expensive from the computational point of view.

In Figure 7, we present the results after 200 iterations of the RFMP_NL for this combination for the optimal regularization parameter. This figure is analogous to Figure 4, which we presented for the first numerical example.

Considering the development of the approximation error and the residual throughout the iteration in Figure 7A, we observe again that the relative residual drops rapidly in the first few iterations to a value of $0.7 \%$, which is below the noise level. The relative approximation error attains a value of $3.10 \%$ after 200 iterations. This is again a very good result if one considered that the data were equipped with a noise level of $1 \%$.

The approximation itself in Figure 7B and the approximation error in Figure 7C are larger in those areas, where the local structures of the solution can be found. In particular, the maximal error is located in the centers of the Abel-Poisson kernels that are present in the prescribed solution. Interestingly, in contrast to the first example, we do not see such a big 


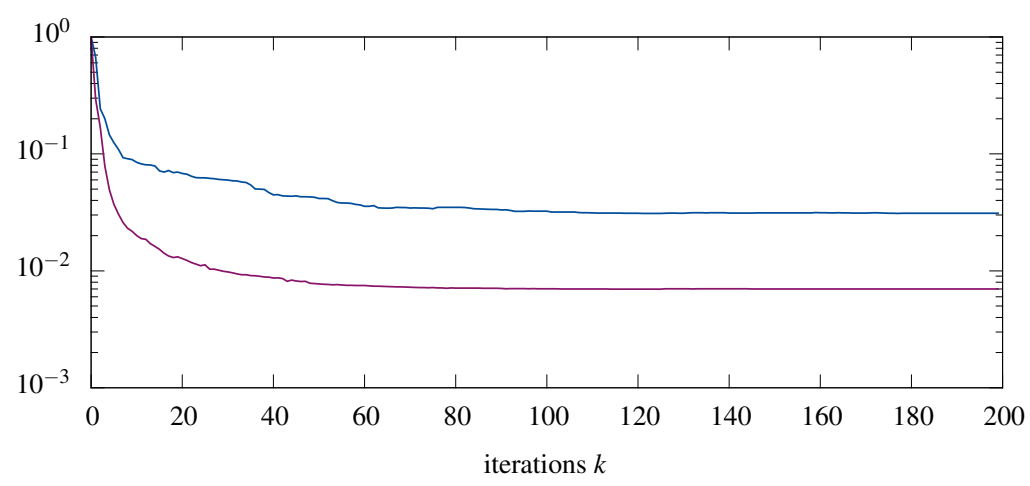

(a) Development of the relative residual (purple) and the relative approximation error (blue) during the iteration of the RFMP_NL.

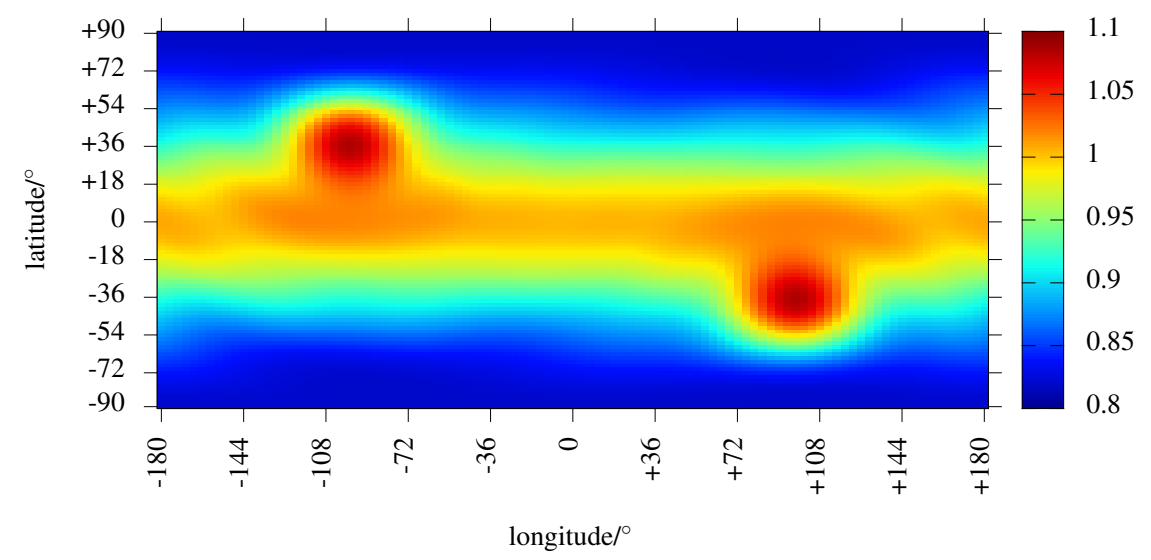

(b) Approximation generated by the RFMP_NL.

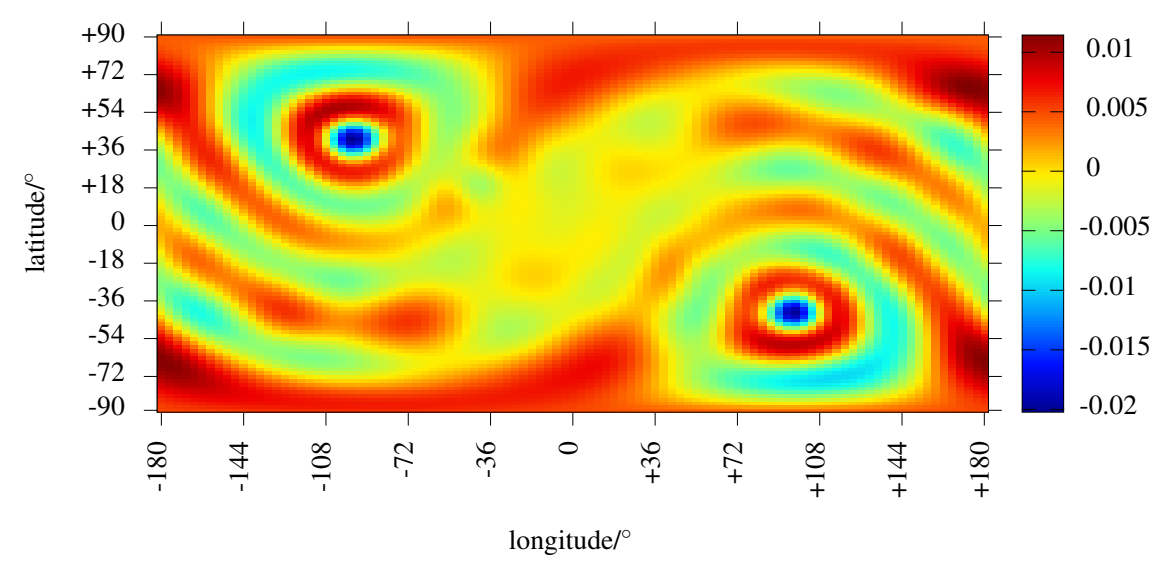

(c) Difference of the solution and the approximation generated by the RFMP_NL.

Figure 7: Results from the application of the RFMP_NL in Example 2. 


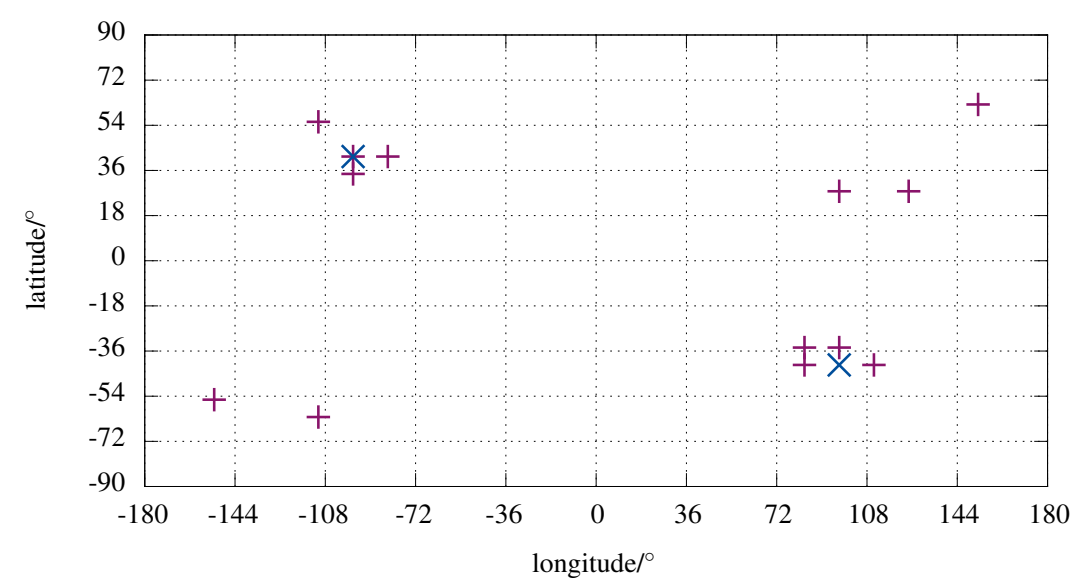

Figure 8: Plot of the centers of the Abel-Poisson kernels that are present in the solution (blue) and the approximation (purple).

amount of artefacts in the error. A look at the absolute values of the pointwise approximation error again shows that the obtained results are very good, since it never exceeds 0.02 , whereas the surface of the body of mass is between 0.8 and 1.1 units away from the center.

If one considers the dictionary elements that were chosen by the RFMP_NL, we observe that the algorithm chose spherical harmonics in 187 of the iterations and Abel-Poisson kernels in only 13 iterations. The algorithm can choose functions from the dictionary multiple times such that the solution consisted of only 89 distinct spherical harmonics and 13 distinct Abel-Poisson kernels. We displayed the centers of these Abel-Poisson kernels in Figure 8 alongside the centers of the kernels that are present in the solution. We observe that 8 of the 13 chosen kernels have their centers near to the kernels of the solution. One of the two kernels in the solution is even chosen itself. Due to the use of noisy data, also some kernels are chosen that are centered where there is no kernel in the solution.

In conclusion, we can say that also in this example, the RFMP_NL produces very good approximations of the prescribed solution. In the derivation of the algorithm, we stated that it will be possible to combine different types of basis functions. The presented example shows that this is indeed true in practice and the results are very promising due to the low approximation errors.

\section{Conclusions and outlook}

In this paper, we derived the Regularized Functional Matching Pursuit for nonlinear inverse problems (RFMP_NL), which is a greedy-type algorithm for nonlinear inverse problems, and applied it to the nonlinear inverse gravimetric problem. For this nonlinear inverse problem, we stated the differences in comparison to the linear inverse gravimetric problem from the theoretical point of view and provided the Fréchet derivative of the associated nonlinear integral operator. Finally, we have applied the RFMP_NL to two synthetic examples, incorporating both only global as well as global and local structures in the prescribed solutions. Empirically, it can be seen that the algorithm shows a convergent behavior in both of the ex- 
amples and that it also combines diverse types of basis functions to build a solution, which is what the RFMP also did for linear inverse problems.

Consequently, the RFMP_NL seems to be a suitable algorithm for the nonlinear inverse gravimetric problem. Due to the high computational effort that comes with the implementation of the algorithm, currently, this is only a proof of concept that the algorithm successfully solves the inverse problem, since the computation time for the presented small examples is already around 9 hours on a node with 12 cores. In the future, we want to apply the algorithm to realistic data sets, for example, data from satellite missions. On the one hand, with rising computer power, we may be able to accomplish this in reasonable time. On the other hand, one could also think about other optimizations of the algorithm to obtain a lower computation time. For example, the strategy that has been used in the derivation of the RWFMP (see Kontak 2018; Kontak and Michel 2018) could also be applied to the RFMP_NL. Additionally, the most expensive part of the algorithm is the re-computation of the Fréchet derivatives in step 2, which is currently done by numerical integration and has to be performed for every dictionary element in every iteration. If one could find a closed formula to analytically compute the integrals, this could also speed up the implementation very much.

Acknowledgements The authors gratefully acknowledge the financial support by the School of Science and Technology of the University of Siegen, Germany. Furthermore, we would like to thank the anonymous reviewer for his valuable comments, which helped to improve the paper.

\section{References}

Amna I, Michel V (2017) Pseudodifferential operators, cubature and equidistribution on the 3D-ball. Numer Funct Anal Optim 38:891-910

Bakushinsky A (1992) The problem of the convergence of the iteratively regularized Gauss-Newton method. Comput Math Math Phys 32:1353-1359

Berkel P, Fischer D, Michel V (2011) Spline multiresolution and numerical results for joint gravitation and normal-mode inversion with an outlook on sparse regularisation. Int J Geomath 1:167-204

Bolton S, Levin S, Bagenal F (2017) Juno's first glimpse of Jupiter's complexity. Geophys Res Lett 44:76637667, special Section "Early Results: Juno at Jupiter"

Burger M, Osher S (2005) A survey on level set methods for inverse problems and optimal design. European J Appl Math 16:263-301

Cea J (1978) Lectures on Optimization-Theory and Algorithms. Springer, Berlin, Heidelberg, New York

Clauser C (2014) Einführung in die Geophysik. Springer, Berlin, Heidelberg

Dagum L, Menon R (1998) OpenMP: an industry-standard API for shared-memory programming. IEEE Comp Sci Eng 5:46-55

Drinkwater M, Floberghagen R, Haagmans R, Muzi D, Popescu A (2003) GOCE: ESA's first Earth explorer core mission. Space Sci Rev 108:419-432

Driscoll J, Healy D (1994) Computing Fourier transforms and convolutions on the 2-sphere. Adv Appl Math 15:202-250

Dziewonski A, Anderson D (1981) Preliminary Reference Earth Model. Phys Earth Planet Inter 25:297-356

Elschner J, Yamamoto M (2006) Uniqueness in determining polygonal sound-hard obstacles with a single incoming wave. Inverse Probl 22:355-364

Engl H, Hanke M, Neubauer A (1996) Regularization of Inverse Problems. Kluwer, Dordrecht

Fischer D (2011) Sparse Regularization of a Joint Inversion of Gravitational Data and Normal Mode Anomalies. PhD thesis, Geomathematics Group, University of Siegen, published by Dr. Hut, München and online at http://dokumentix.ub.uni-siegen.de/opus/volltexte/2012/544/

Fischer D, Michel V (2012) Sparse regularization of inverse gravimetry-case study: spatial and temporal mass variations in South America. Inverse Probl 28:065012

Fischer D, Michel V (2013a) Automatic best-basis selection for geophysical tomographic inverse problems. Geophys J Int 193:1291-1299

Fischer D, Michel V (2013b) Inverting GRACE gravity data for local climate effect. J Geod Sci 3:151-162 
Flechtner F, Morton P, Webb F (2014) Status of the GRACE follow-on mission. In: Marti U (ed) Gravity, Geoid and Height Systems, Springer, Cham, pp 117-121

Freeden W, Gerhards C (2013) Geomathematically Oriented Potential Theory. CRC Press, Boca Raton

Freeden W, Michel V (2004) Multiscale Potential Theory. With Applications to Geoscience. Birkhäuser, Boston, Basel, Berlin

Freeden W, Gervens T, Schreiner M (1998) Constructive Approximation on the Sphere. Oxford University Press, Oxford

Galassi M, Davies J, Theiler J, Gough B, Jungman G, Alken P, Booth M, Rossi F (2009) GNU Scientific Library Reference Manual, 3rd edn. Network Theory, Bristol

Gutting M, Kretz B, Michel V, Telschow R (2017) Study on parameter choice methods for the RFMP with respect to downward continuation. Front Appl Math Stat 3:Article 10

Hanke M, Neubauer A, Scherzer O (1995) A convergence analysis of the Landweber iteration for nonlinear ill-posed problems. Numer Math 72:21-37

Hettlich F, Rundell W (1996) Iterative methods for the reconstruction of an inverse potential problem. Inverse Probl 12:251-266

Hettlich F, Rundell W (1997) Recovery of the support of a source term in an elliptic differential equation. Inverse Probl 13:959-976

Hettlich F, Rundell W (2000) A second degree method for nonlinear inverse problems. SIAM J Numer Anal 37:587-620

Holmes M (2009) Introduction to the Foundations of Applied Mathematics. Springer, New York

Isakov V (1990) Inverse Source Problems. American Mathematical Society, Providence

Isakov V (2006) Inverse Problems for Partial Differential Equations, 2nd edn. Springer, New York

Kaltenbacher B, Neubauer A, Scherzer O (2008) Iterative Regularization Methods for Nonlinear Ill-Posed Problems. de Gruyter, Berlin

Kerningham B, Ritchie D (1988) The C Programming Language, 2nd edn. Prentice Hall, Englewood Cliffs

Kontak M (2018) Novel Algorithms of Greedy-type for Probability Density Estimation as well as Linear and Nonlinear Inverse Problems. PhD thesis, Geomathematics Group, University of Siegen, published online at http://dokumentix.ub.uni-siegen.de/opus/volltexte/2018/1316/

Kontak M, Michel V (2018) The regularized weak functional matching pursuit for linear inverse problems. Preprint Submitted for publication

Landweber L (1951) An iteration formula for Fredholm integral equations of the first kind. Amer J Math 73:615-624

Lauricella G (1912) Sulla distribuzione della massa nell'interno dei pianeti. Rend Acc Linei XXI:18-26

Levenberg K (1944) A method for the solution of certain non-linear problems in least squares. Quart Appl Math 2:164-168

Leweke S, Michel V, Telschow R (2018) On the non-uniqueness of gravitational and magnetic field data inversion (survey article). In: Freeden W, Nashed M (eds) Handbook of Mathematical Geodesy, Birkhäuser, pp 883-919

Marquardt D (1963) An algorithm for least-squares estimation of nonlinear parameters. J Soc Indust Appl Math 11:431-441

Matousek S (2007) The Juno new frontiers mission. Acta Astronautica 61:932-939

Michel V (2013) Lectures on Constructive Approximation. Fourier, Spline, and Wavelet Methods on the Real Line, the Sphere, and the Ball. Birkhäuser, New York

Michel V (2015) RFMP: an iterative best basis algorithm for inverse problems in the geosciences. In: Freeden W, Nashed M, Sonar T (eds) Handbook of Geomathematics, 2nd edn, Springer, Berlin, Heidelberg, pp 2121-2147

Michel V, Fokas A (2008) A unified approach to various techniques for the non-uniqueness of the inverse gravimetric problem and wavelet-based methods. Inverse Probl 24:045019

Michel V, Orzlowski S (2017) On the convergence theorem for the Regularized Functional Matching Pursuit (RFMP) algorithm. Int J Geomath 8:183-190

Michel V, Telschow R (2014) A non-linear approximation method on the sphere. Int J Geomath 5:195-224

Michel V, Telschow R (2016) The Regularized Orthogonal Functional Matching Pursuit for ill-posed inverse problems. SIAM J Numer Anal 54:262-287

Mikhlin S (1970) Mathematical Physics, An Advanced Course. North-Holland, Amsterdam, London

Novikov P (1938) Sur le problème inverse du potentiel. Dokl Akad Nauk 18:165-168

OpenMP Architecture Review Board (ed) (2013) OpenMP Application Program Interface. Version 4.0, URL: http://www.openmp.org/specifications/

Pizzetti P (1909) Corpi equivalenti rispetto alla attrazione newtoniana esterna. Rom Acc L Rend XVIII:211215 
Pizzetti P (1910) Intorno alla possibli distribuzioni della massa nell'interno della terra. Annali di Mat, Milano XVII:225-258

Reigber C, Schwintzer P, Lühr H (1999) The CHAMP geopotential mission. Boll Geof Teor Appl 40:285-289

Rieder A (2003) Keine Probleme mit inversen Problemen. Vieweg, Wiesbaden

Tapley B, Bettadpur S, Watkins M, Reigber C (2004) The gravity recovery and climate experiment: Mission overview and early results. Geophys Res Lett 31:L09607

Telschow R (2014) An Orthogonal Matching Pursuit for the Regularization of Spherical Inverse Problems. $\mathrm{PhD}$ thesis, Geomathematics Group, University of Siegen, published 2015 by Dr. Hut, München

Tikhonov A, Glasko V (1965) Use of the regularization method in non-linear problems. USSR Comp Math Math Phys 5:93-107

Wald A, Schuster T (2017) Sequential subspace optimization for nonlinear inverse problems. J Inverse IllPosed Probl 25:99-117

Weck N (1972) Inverse Probleme der Potentialtheorie. Appl Anal 2:195-204

Yosida K (1980) Functional Analysis, 6th edn. Springer, Berlin, Heidelberg, New York

Zuber M, Smith D, Lehman D, Hoffmann T, Asmar S, Watkins M (2013) Gravity recovery and interior laboratory (GRAIL): mapping the lunar interior from crust to core. Space Sci Rev 178:3-24 